\title{
Energy conserving balance of explicit time steps to combine implicit and explicit algorithms in structural dynamics
}

\author{
L. Noels ${ }^{1}$, L. Stainier ${ }^{2}$, J.-P. Ponthot* \\ Université de Liège, LTAS-Milieux Continus \& Thermomécanique, Chemin des Chevreuils 1, B-4000 Liège, Belgium
}

\begin{abstract}
Recent developments have proved the advantage of combining both time implicit and time explicit integration algorithms in structural dynamics. A major problem is to define the initial conditions for the implicit simulation on the basis of a solution obtained from an unbalanced explicit resolution. The unbalanced nature of the explicit algorithm leads to oscillations in the fields of interest. Therefore, the values obtained after an explicit computation cannot be used directly as initial conditions for an implicit simulation. In this paper, we develop such initial values that lead to a stable (no numerical creation of energy) and energy-conserving transition.
\end{abstract}

Keywords: Structural dynamics; Implicit; Explicit; Time integration; Energy conserving

\section{Introduction}

A current problem in computational dynamics is to be able to simulate numerically the response of a very non-linear structure submitted to dynamic loading. The finite-element model, resulting from the spatial discretization of the balance equations describing these dynamic problems, needs to be temporally integrated with a step by step approach. The time-integration algorithms can be separated in two families: the explicit algorithms family [1-5], and the implicit algorithms family [1-3,5-8]. When studying the numerical properties of an algorithm such as that of Newmark's scheme [6], it appears that, for a linear system, the unconditional (i.e. for any time step size) stability (i.e. no numerical energy is added to the energy of the

\footnotetext{
* Corresponding author. Tel.: +32 4 3669310; fax: +32 43669141.

E-mail address: jp.ponthot@ulg.ac.be (J.-P. Ponthot).

${ }^{1}$ Research Fellow at the Belgian National Fund for Scientific Research (FNRS).

${ }^{2}$ Research Associate at the Belgian National Fund for Scientific Research (FNRS).
} 
system) requires that the parameters of the algorithm verify some relations. Once these relations are verified, it appears that the resulting equations have to be iteratively resolved. The algorithm is therefore called implicit and the balance equation is solved, for example, with a Newton-Raphson scheme. To avoid these iterations that are very time consuming, especially for non-linear systems, the parameters of the algorithm can be chosen so that the balance equations need not to be iteratively solved, but are used to predict the accelerations. This explicit solution is not able to verify the relations that lead to an unconditionally stable algorithm. Indeed, a stability limit does exist. As long as the time step size is lower than a critical value, the algorithm is stable. But if the time step size becomes larger, the numerical integration adds energy to the system, leading to an unstable integration.

Explicit methods are therefore generally used to simulate fast dynamics and highly non-linear simulations with a large number of degrees of freedom. For such a model, if one uses an implicit algorithm, the iterations are very expensive and the problems of convergence are frequent [9]. Moreover, in order to correctly capture the physics of fast dynamics problems, small time steps have to be used. But if the problem is governed by low frequencies, the limitation on the time step size leads to a very important number of time steps. The fact that the balance equations are not verified at each time step can also lead to a loss of accuracy in the integration. On the other hand, an implicit simulation has the advantages of requiring less time steps, while being more accurate [9-12]. Let us note that for some clearly fast dynamics problems, implicit solutions can be cheaper and more accurate than explicit solutions $[13,14]$.

Moreover, many practical problems combine some time intervals ideally integrated by explicit methods and some other time intervals ideally integrated by implicit methods. For example, a stamping process is highly non-linear and, though essentially a quasi-static process, is thus generally integrated with an explicit algorithm. But the spring-back process occurring after the stamping itself when the loads are removed, has to be integrated by an implicit algorithm to be accurate [9-11]. A methodology combining both types of algorithms was used by Finn et al. [15] and by Narkeeran and Lovell [16]. In their approach, when shifting from an explicit algorithm to an implicit one, the velocities and the accelerations are set to zero. Nevertheless, these conditions of transition cannot be used for problems where the dynamics is much more complicated. For example, when studying a blade loss in an aircraft engine, we want the blade impact on the casing to be computed by an explicit scheme, but the time interval after the impact, when the rotational velocity of the engine is decreased, should be computed with an implicit algorithm. If, for such a simulation, the velocities and the accelerations are set to null, the dynamics of the simulation is completely modified by this artefact, leading to a wrong solution. Moreover, the unbalanced nature of the explicit algorithm leads to oscillations in the simulation. Therefore, the values after an explicit computation cannot be used as initial conditions of an implicit simulation. Thus, in a previous work [17], when the decision to switch from an explicit algorithm to an implicit one has been taken, we have proposed to stabilize the last explicit steps to be able to start an implicit simulation with stabilized initial values. We have applied this method to impact simulations [18] and to sheet metal forming simulations [19]. Nevertheless, if this method seemed to be stable and accurate, we were not able to prove it mathematically. Using the recent considerations initiated by Simo and Tarnow [20] on the stability and the thermodynamical consistency of implicit algorithms in the non-linear range, we propose in this work to develop, in the fully non-linear range, a stable and thermodynamically consistent methodology that balances explicit steps. The balance method we propose to establish gives the initial conditions to carry on a process with an implicit scheme without loss of accuracy.

Let us note that when switching from an implicit method to an explicit one, the explicit simulation starts with a balanced solution, thus leading to no stability problems (other than the stability problems inherent from the use in the non-linear range of an explicit scheme that is proved to be stable only in the linear range). Such a shift to an explicit method was used by Jung and Yang [21] who proposed, in sheet metal forming simulations, to start the computation with an implicit scheme and then to shift to an explicit one when convergence problems appear. We used such a shift in $[17,18]$ for structures submitted to impact loading. Finally, Rust and Schweizerhof [12] proposed to shift from an implicit algorithm to an explicit one to 
compute the limit load and buckling mode of a thin-walled structure. In their approach, the structure is loaded with an implicit algorithm while this load remains lower than the limit load. During the loading phase, the implicit method has the advantage of using large time steps and being balanced, contrarily to the explicit one. But when the structure is close to buckling, and during the buckling phase, the non-linearities cause the explicit algorithm to be more efficient.

The outline of the paper is as follows. Section 2 presents the problem of time integration. After an introduction to the notations and balance equations, we will briefly recall the explicit scheme based on a central difference method, that can be proved to be stable only in the linear range. Next, we will present the implicit Energy Momentum Conserving Algorithm (EMCA), first proposed by Simo and Tarnow [20]. This algorithm conserves the linear momentum, the angular momentum, and the energy of an isolated system in the non-linear range. But these conservation properties are reached only if the internal forces of the system are "well designed". We will recall this expression of the forces for a mass-spring system and for an elasto-plastic material model. Thanks to the previous expressions, when the decision to switch from an explicit algorithm to an implicit algorithm has been taken, we will show in Section 3 how to balance the explicit steps in order to obtain stable initial conditions to start the implicit simulation. We will use the last explicit steps as initial values for a subsequent implicit step of larger size. Studying the predictor-corrector algorithms, and adapting the expressions of the internal forces, we will demonstrate that our proposed balancing algorithm is stable (no numerical energy is introduced), conserves the linear momentum, the angular momentum and the energy in the non-linear range (i.e. that it is thermodynamically consistent). Some numerical examples will be presented in Section 4. First, we will use our balance method to perform a long-duration simulation of a non-linear mass-spring system. Since this computation will be shown to occur without numerical introduction of any energy, it will demonstrates numerically that our method is stable in the non-linear range. Since our balance method is developed to be able to shift from an explicit algorithm to an implicit one without introducing numerical energy in the system, we will present two elasto-plastic dynamics problems where the integration method will shift from a method to another one. When shifting to an implicit algorithm, the last explicit steps will be balanced with our proposed method. These examples will show that the proposed shift method allows the implicit simulation, following an explicit one, to be reached without loss of accuracy.

\section{Time integration}

In this section, after having explained our notations, we recall the form of the balance equations. Then, we present how to integrate these equations in time, with an explicit algorithm or with an implicit algorithm. For both methods, the stability of the integration is discussed in the linear and in the non-linear range.

\subsection{Notations}

Let $V \subset \mathbb{R}^{3}$ be the volume of the system, and $S$ the current surface bounding $V$. Since our developments are conducted for arbitrary strains, we have to distinguish between (at least) two configurations: the reference configuration at time 0 and the current one at time $t$. Both configuration can differ significantly by an arbitrary deformation which is not restricted in magnitude. The initial density is denoted $\rho_{0}: V_{0} \rightarrow \mathbb{R}_{+}$. Let us use a subscript 0 to indicate a value in the reference configuration. Volume $V$ is discretized in finite elements and each point of $V$ is referenced by its initial positions $\vec{x}_{0} \in \mathbb{R}^{3}$. The surface is decomposed into a part $S_{\vec{x}}$ where the displacements are imposed and a part $S_{\vec{t}}$ where the loads are imposed. We always have $S_{\vec{x}} \cup S_{\vec{t}}=S$ and $S_{\vec{x}} \cap S_{\vec{t}}=0$. Let $\vec{x}$ be the current positions mapping. The two-point gradient of deformation tensor is defined by 


$$
\mathbf{F} \equiv \frac{\partial \vec{x}}{\partial \vec{x}_{0}}
$$

Its inverse is denoted by $\mathbf{f}$ and its determinant by $J=\operatorname{det} \mathbf{F}$. The set $\mathscr{X}$ of admissible positions is defined by

$$
\mathscr{X} \equiv\left\{\vec{x}: V_{0} \rightarrow \mathbb{R}^{3} \mid\left[J>0 \text { and }\left.\vec{x}\right|_{S_{\vec{x}}}=\overrightarrow{\vec{x}}\right] \quad \forall \vec{x}_{0} \in V_{0}\right\},
$$

where $\overline{\vec{x}}$ are the imposed positions and $V_{0}$ the reference volume.

Let $t$ be the time and $\mathscr{T}=\left[0, t_{f}\right]$ be the time interval of integration. Therefore the motion is a map defined by $t \in \mathscr{T} \rightarrow \vec{x}(t) \in \mathscr{X}$. During this motion, the volume is loaded by the body forces $\vec{b}(t)$ : $V_{0} \times \mathscr{T} \rightarrow \mathbb{R}^{3}$. If $\boldsymbol{\Sigma}$ is the Cauchy stress tensor, the surface loads $\vec{t}(t): S_{\overrightarrow{t_{0}}} \times \mathscr{T} \rightarrow \mathbb{R}^{3}$ have to verify $\vec{t}(t)=\boldsymbol{\Sigma}(t) \vec{n}(t)$ with $\vec{n}$ the current unit outward normal of $S$.

The finite element decomposition [2] is reached by the use of shape functions $\varphi^{\xi}: V_{0} \rightarrow \mathbb{R}$ with $\xi \in\left[1, n_{\text {nodes }}\right]$ ( $n_{\text {nodes }}$ is the number of nodes), with $\varphi^{\xi}\left(\vec{x}_{0}^{\mu}\right)=\delta_{\xi}^{\mu}$ (with $\delta$ the Kronecker symbol). It leads, for $\xi \in\left[1, n_{\text {nodes }}\right]$, to

$$
\begin{aligned}
& \vec{x}\left(\vec{x}_{0}\right)=\varphi^{\xi}\left(\vec{x}_{0}\right) \vec{x}^{\xi}, \\
& \dot{\vec{x}}\left(\vec{x}_{0}\right)=\varphi^{\xi}\left(\vec{x}_{0}\right) \dot{\vec{x}}^{\xi}, \\
& \ddot{\vec{x}}\left(\vec{x}_{0}\right)=\varphi^{\xi}\left(\vec{x}_{0}\right) \ddot{\vec{x}}^{\xi},
\end{aligned}
$$

where Einstein's notations have been used.

\subsection{The balance equations}

Using notations of Section 2.1, the balance equation at node $\xi$ leads to

$$
M^{\xi \mu}[\ddot{\vec{x}}]^{\mu}=\left[\vec{F}_{\text {ext }}-\vec{F}_{\text {int }}\right]^{\xi}
$$

with $M_{\vec{\xi}}^{\xi \mu}$ the mass matrix component relative to nodes $\xi$ and $\mu$, with $\vec{F}_{\text {ext }}^{\xi}$ the external forces at node $\xi$, and with $\vec{F}_{\text {int }}^{\xi}$ the internal forces at node $\xi$. The external forces have the following expression

$$
\vec{F}_{\text {ext }}^{\xi}=\int_{V_{0}}\left\{\rho_{0} \vec{b} \varphi^{\xi}\right\} \mathrm{d} V_{0} \cdot \delta \vec{x}^{\xi}+\int_{S_{\vec{t}}}\left\{\vec{t} \varphi^{\xi}\right\} \mathrm{d} S
$$

and the internal forces can be written as

$$
\vec{F}_{\text {int }}^{\xi}=\int_{V_{0}}\left\{\boldsymbol{\Sigma}^{\mathrm{T}} \mathbf{f}^{\mathrm{T}} \vec{D}^{\xi} J\right\} \mathrm{d} V_{0},
$$

where $\vec{D}$ is the derivative of the shape functions (in the reference configuration, i.e. $\vec{D}^{\xi}=\frac{\partial \varphi^{\xi}}{\partial \vec{x}_{0}}$ ).

For an exact solution, Eq. (2.4) has to be verified for every time $t \in \mathscr{T}$. To integrate this equation in time, the time interval $\mathscr{T}$ is decomposed into some partitions $\left[t^{n}, t^{n+1}\right]$ (such that $\mathscr{T}=\bigcup_{n=0}^{N}\left[t^{n}, t^{n+1}\right]$ ) with $\Delta t=t^{n+1}-t^{n}$ the time step size. Therefore, superscripts $n$ and $n+1$ are respectively used to denote a value at time $t^{n}$ and at time $t^{n+1}$.

\subsection{Particular case of the rod}

Eq. (2.6) defines the general form of a three dimensional problem. The formulation for a discretized rod can be derived from it. Let $A_{0}$ be the (constant) initial section of the rod and $l$ be its length at time $t$. Therefore, introducing $E$, the Young's modulus, the expression for internal forces defined by Eq. (2.6), with first order shape functions, becomes 


$$
\begin{aligned}
& \vec{F}_{\text {int }}^{\xi}=\frac{k\left[l-l_{0}\right]}{l}\left[\vec{x}^{\xi}-\vec{x}^{\mu}\right], \\
& \vec{F}_{\text {int }}^{\mu}=\frac{k\left[l-l_{0}\right]}{l}\left[\vec{x}^{\mu}-\vec{x}^{\xi}\right]
\end{aligned}
$$

with $k=\frac{E A_{0}}{l_{0}}, l=\left\|\vec{x}^{\mu}-\vec{x}^{\xi}\right\|$ and $\mu$ and $\xi$ the indices of the two end nodes. Defining $U_{\text {int }}=\frac{1}{2} k\left[l-l_{0}\right]^{2}$ the internal potential, the rod can be seen as a spring with

$$
\begin{aligned}
& \vec{F}_{\text {int }}^{\xi}=\frac{\partial U_{\text {int }}(l)}{\partial \vec{x}^{\xi}}, \\
& \vec{F}_{\text {int }}^{\mu}=\frac{\partial U_{\text {int }}(l)}{\partial \vec{x}^{\mu}} .
\end{aligned}
$$

With $m=\frac{\rho_{0} A_{0} l_{0}}{2}$, masses at nodes $\xi$ and $\mu$ can be written as

$$
M^{\xi \mu}=m \delta_{\xi \mu} \text {. }
$$

\subsection{Explicit time integration of the balance equation}

The explicit scheme most commonly used to integrate Eq. (2.4) in time is the central difference scheme $[1,2]$. At time $t^{n+1}$ the solution depends only on the solution at time $t^{n}$ and not on solution at time $t^{n+1}$. Therefore, no iteration is needed to integrate Eq. (2.4).

First, knowing the solution of Eq. (2.4) at time $t^{n}$ (i.e. $\vec{x}^{n}, \dot{\vec{x}}^{n-\frac{1}{2}}, \ddot{\vec{x}}^{n}$ are supposed to be known as well as the stress tensor and associated values), velocities are computed at time $t^{n+\frac{1}{2}}$ for each node $\xi$

$$
\left[\dot{\vec{x}}^{n+\frac{1}{2}}\right]^{\xi}=\left[\dot{\vec{x}}^{n-\frac{1}{2}}\right]^{\xi}+\Delta t\left[\ddot{\vec{x}}^{n}\right]^{\xi}
$$

Positions at time $t^{n+1}$ are deduced from these velocities

$$
\left[x^{n+1}\right]^{\xi}=\left[x^{n}\right]^{\xi}+\Delta t\left[\dot{x}^{n+\frac{1}{2}}\right]^{\xi} .
$$

Finally, the balance Eq. (2.4) is used in an explicit way to evaluate the accelerations at time $t^{n+1}$

$$
\left[\ddot{\vec{x}}^{n+1}\right]^{\xi}=\left[M^{-1}\right]^{\xi \mu}\left[\vec{F}_{\text {ext }}^{n+1}-\vec{F}_{\text {int }}^{n+1}\right]^{\mu} .
$$

The mass matrix is lumped to improve the efficiency of the algorithm. The internal forces are computed from Eq. (2.7) for a mass-spring system and from Eq. (2.6) for a general 3D elasto-viscoplastic model.

The stability of such an explicit algorithm can only be studied for a linear model. Let us assume the following relation between the velocities in the configuration $n+\frac{1}{2}$ and in the configuration $n+1$ (i.e. constant time step)

$$
\left[\dot{\vec{x}}^{n+\frac{1}{2}}\right]^{\xi} \equiv\left[\dot{\vec{x}}^{n+1}\right]^{\xi}-\frac{\Delta t}{2}\left[\ddot{\vec{x}}^{n+1}\right]^{\xi} .
$$

Then, system of Eqs. (2.10), (2.11) and (2.12) can be shown to be equivalent to the Newmark scheme for some particular choice of parameters [2]. Therefore the algorithm is stable if and only if

$$
\Delta t \leqslant \frac{2}{\omega_{\max }}
$$

with $\omega_{\max }$ the maximal pulsation of the system. Considering a security factor $\gamma_{s}<1$ to take into account the non-linearities of the system, Eq. (2.14) becomes 


$$
\Delta t=\frac{2 \gamma_{s}}{\omega_{\max }} .
$$

The maximal pulsation of the system can be evaluated as proposed by Flanagan and Belytschko [22] or more efficiently through the power iteration method as proposed by Benson [5]. We have adopted the latter solution here. The security parameter can be given as a fixed value or better evaluated by an estimation on the integration error $[23,24]$ in order to be able to integrate more efficiently the resulting non-linear system.

\subsection{Implicit time integration of the balance equation}

The solution proposed by Simo and Tarnow [20] to be able to obtain an integration algorithm that is stable and thermodynamically consistent in the non-linear range is to adapt the mid-point scheme (see principle below) for each kind of non-linearity under interest.

\subsubsection{Basic principle}

In the mid-point scheme, the relation between the positions and velocities is given by

$$
\left[\vec{x}^{n+1}\right]^{\xi}=\left[\vec{x}^{n}\right]^{\xi}+\frac{\Delta t}{2}\left[\dot{\vec{x}}^{n+1}\right]^{\xi}+\frac{\Delta t}{2}\left[\dot{\vec{x}}^{n}\right]^{\xi},
$$

while the relation between the velocities and accelerations is

$$
\left[\dot{\vec{x}}^{n+1}\right]^{\xi}=\left[\dot{\vec{x}}^{n}\right]^{\xi}+\frac{\Delta t}{2}\left[\ddot{\vec{x}}^{n+1}\right]^{\xi}+\frac{\Delta t}{2}\left[\ddot{\vec{x}}^{n}\right]^{\xi} .
$$

The balance Eq. (2.4) is implicitly solved at node $\xi$ through the relation

$$
\frac{1}{2} M^{\xi \mu}\left[\ddot{\vec{x}}^{n+1}+\ddot{\vec{x}}^{n}\right]^{\mu}=\left[\vec{F}_{\mathrm{ext}}^{n+\frac{1}{2}}-\vec{F}_{\mathrm{int}}^{n+\frac{1}{2}}\right]^{\xi},
$$

where $\vec{F}^{n+\frac{1}{2}}$, is the expression of the forces (internal or external) in the configuration $n+\frac{1}{2}$ (i.e. at time $t^{n+\frac{1}{2}}$ ). In the classical mid-point scheme, this expression is computed from Eq. (2.6) or from Eq. (2.7) evaluated at position $\frac{\vec{x}^{n+1}+\vec{x}^{n}}{2}$ and not at positions $\vec{x}^{n+1}$. But this expression must be designed in order to achieve an integration algorithm that is consistent in the non-linear range (i.e. the conservation laws have to be verified).

\subsubsection{The conservation laws}

Assuming pure Neumann boundary condition (i.e. $S_{\vec{x}}=0$ ), during the time interval of interest, the motion of the center of gravity must depend only on the external forces. Moreover, the modification of the energy of the system must depend only on these external forces too. Thus, the variation of the energy of the system must be equal to the work done by the external forces minus the internal dissipation (due to plasticity, frictional sliding, ...) of the system. If these conditions are satisfied, the integration is called thermodynamically consistent [20]. To reach this goal, internal forces have to verify some conditions that we will give below. For more details, we refer the reader to [20].

Conservation of the linear momentum $\vec{L}$ is reached if

$$
\vec{L}^{n+1}-\vec{L}^{n}=\sum_{\xi} M^{\xi \mu}\left[\dot{\vec{x}}^{n+1}-\dot{\vec{x}}^{n}\right]^{\mu}=\Delta t \sum_{\xi}\left[\vec{F}_{\mathrm{ext}}^{n+\frac{1}{2}}\right]^{\xi} .
$$

This relation is verified if the internal forces verify

$$
\sum_{\xi}\left[\vec{F}_{\text {int }}^{n+\frac{1}{2}}\right]^{\xi}=0 .
$$


Conservation of the angular momentum $\vec{J}$ is reached if

$$
\vec{J}^{n+1}-\vec{J}^{n}=M^{\xi \mu}\left[\vec{x}^{\xi} \wedge \dot{\vec{x}}^{\mu}\right]^{n+1}-M^{\xi \mu}\left[\vec{x}^{\xi} \wedge \dot{\vec{x}}^{\mu}\right]^{n}=\Delta t\left[\frac{\vec{x}^{n}+\vec{x}^{n+1}}{2}\right]^{\xi} \wedge\left[\vec{F}_{\text {ext }}^{n+\frac{1}{2}}\right]^{\xi}
$$

with the operation $\vec{a} \wedge \vec{b}$ denoting the vector product of $\vec{a}$ and $\vec{b}$. To verify Eq. (2.21), internal forces have to verify

$$
\left[\frac{\vec{x}^{n+1}+\vec{x}^{n}}{2}\right]^{\xi} \wedge\left[\vec{F}_{\text {int }}^{n+\frac{1}{2}}\right]^{\xi}=0
$$

Let $E, U_{\text {int }}, W_{\text {ext }}$ and $K$ be respectively the total energy of the system, the internal energy, the work done by the external forces and the kinetic energy. Conservation of the energy $E \equiv K+U_{\text {int }}$ is discretized on a time step by

$$
E^{n+1}-E^{n}=W_{\mathrm{ext}}^{n+1}-W_{\mathrm{ext}}^{n}-\Delta_{\mathrm{int}},
$$

where $\Delta_{\mathrm{int}} \geqslant 0$ is the internal dissipation, during the step, coming from the irreversible behavior of the material, the frictional sliding,... Therefore, to verify Eq. (2.23), the relation that the internal forces have to verify is

$$
\left[\vec{F}_{\text {int }}^{n+\frac{1}{2}}\right]^{\xi} \cdot\left[\vec{x}^{n+1}-\vec{x}^{n}\right]^{\xi}=U_{\text {int }}^{n+1}-U_{\text {int }}^{n}+\Delta_{\text {int }} .
$$

Now in order to obtain a scheme consistent with thermodynamics, the expression of the internal forces has to be designed to verify Eqs. (2.20), (2.22) and (2.24).

\subsubsection{Construction of the internal forces}

The internal force expression consistent with thermodynamics was given for a spring by Simo et al. [25], and by Gonzalez and Simo [26,27]. To be able to verify the Eqs. (2.20), (2.22) and (2.24) Eq. (2.7) is rewritten as

$$
\begin{aligned}
{\left[\vec{F}_{\text {int }}^{n+\frac{1}{2}}\right]^{\xi}=} & \frac{U\left(l^{n+1}\right)-U\left(l^{n}\right)}{l^{n+1^{2}}-l^{n^{2}}}\left\{\left[\vec{x}^{n+1}+\vec{x}^{n}\right]^{\xi}-\left[\vec{x}^{n+1}+\vec{x}^{n}\right]^{\mu}\right\} \quad \text { if } l^{n+1} \neq l^{n} \\
= & \frac{\frac{\partial}{\partial l} U\left(\frac{l^{n+1}+l^{n}}{2}\right)}{l^{n+1}+l^{n}}\left\{\left[\vec{x}^{n+1}+\vec{x}^{n}\right]^{\xi}-\left[\vec{x}^{n+1}+\vec{x}^{n}\right]^{\mu}\right\} \quad \text { if } l^{n+1} \rightarrow l^{n} .
\end{aligned}
$$

Conservation of the linear momentum (2.20) and of the angular momentum (2.22) are directly obtained from Eq. (2.25). Conservation of the energy (2.24), with $\Delta_{\text {int }}=0$ (no internal dissipation) is obtained by setting the potential $U$ equal to the internal energy $U_{\text {int }}$.

Simo and Tarnow [20] and Simo and Gonzalez [28] gave a consistent expression for Saint Venant-Kirchhoff hyperelastic materials. A generalization to other hyperelastic models was given by Laursen and Meng [29], who iteratively solved a new equation for each Gauss point to determine the adequate second Piola-Kirchhoff stress tensor. Another solution that avoids this iterative procedure leads to a general formulation of the second Piola-Kirchhoff stress tensor, as given by Gonzalez [30]. This formulation is valid for general hyperelastic materials. The EMCA (Energy Momentum Conserving Algorithm) was recently extended to dynamic finite deformation plasticity by Meng and Laursen [31,32]. In such a formulation, the algorithm remains energy conserving when no irreversible deformation occurs, and "dissipates energy in a manner consistent with the physical model in use" (sic) when plastic deformation occurs. Finally, we have extended in $[33,34]$ the model of the elasto-plastic material to the hypoelastic description. Such a model integrates spatially Cauchy stresses, which do not derive from a potential but are computed form 
a hardening law. In this section we will briefly recall the expression of the internal forces for a hypoelastic bulk model. Our present work that consists to balance the explicit steps can easily be extended to other models such as hyperelastic bulk ones or frictional contact model.

Deformation gradient $\mathbf{F}$ defined in Eq. (2.1) is incrementally computed between the two configurations $n$ and $n+1$. Thanks to the Polar Decomposition Theorem, $\mathbf{F}$ is decomposed into the product of a rotation tensor $\mathbf{R}$ and a positive definite symmetric tensor $\mathbf{U}$ such that

$$
\mathbf{F}_{n}^{n+1}=\frac{\partial \vec{x}^{n+1}}{\partial \vec{x}^{n}}=\mathbf{R}_{n}^{n+1} \mathbf{U}_{n}^{n+1} .
$$

The inverse of $\mathbf{F}$ is denoted by $\mathbf{f}$ and the determinant of $\mathbf{F}$ is denoted by $J$. Green-Lagrange strain tensor $\mathbf{G L}$, Almansi strain tensor $\mathbf{A}$ and natural strain tensor $\mathbf{E}$ are computed from $\mathbf{F}$ respectively as

$$
\begin{aligned}
& \mathbf{G L}_{n}^{n+1}=\frac{1}{2}\left[\mathbf{F}_{n}^{n+1^{\mathrm{T}}} \mathbf{F}_{n}^{n+1}-\mathbf{I}\right], \\
& \mathbf{A}_{n}^{n+1}=\frac{1}{2}\left[\mathbf{I}-\mathbf{f}_{n}^{n+1^{\mathrm{T}}} \mathbf{f}_{n}^{n+1}\right], \\
& \mathbf{E}_{n}^{n+1}=\frac{1}{2} \ln \left[\mathbf{F}_{n}^{n+1^{\mathrm{T}}} \mathbf{F}_{n}^{n+1}\right] .
\end{aligned}
$$

As far as the integration of the hypoelastic constitutive model (for elasto-viscoplastic material models) is concerned, the incremental procedure is the following (see [35] for details). The Cauchy stress are then obtained by

$$
\boldsymbol{\Sigma}^{n+1}=\mathbf{R}_{n}^{n+1}\left[\boldsymbol{\Sigma}^{n}+\mathscr{H}: \mathbf{E}_{n}^{n+1}-\mathbf{s}^{\mathbf{c}}\right] \mathbf{R}_{n}^{n+1^{\mathrm{T}}},
$$

where $\mathscr{H}$ is the four order Hooke tensor and where $\mathbf{s}^{c}$ is the viscoplastic correction obtained by the radial return mapping scheme [35-38] to verify the discretized von Mises criterion evaluated at time $t^{n+1}$. This method guarantees the incremental objectivity of the algorithm. Now that the current state of stress can be computed, one still has to verify the thermodynamic consistency of the time integration scheme.

To be able to satisfy the Eqs. (2.20), (2.22) and (2.24), the expression of the internal forces (2.6) is rewritten as

$$
\begin{aligned}
& {\left[\vec{F}_{\text {int }}^{n+\frac{1}{2}}\right]^{\xi}=\frac{1}{2}\left[\vec{F}_{\text {int }}^{*}+\vec{F}_{\text {int }}^{* *}\right]^{\xi},} \\
& {\left[\vec{F}_{\text {int }}^{*}\right]^{\xi}=\frac{1}{2} \int_{V_{0}}\left\{\left[\mathbf{I}+\mathbf{F}_{n}^{n+1}\right]\left[\mathbf{\Sigma}^{n^{\mathrm{T}}}+\mathbf{C}^{*}\right] \mathbf{f}_{0}^{n^{\mathrm{T}}} \vec{D}^{\xi} J_{0}^{n}\right\} \mathrm{d} V_{0},} \\
& {\left[\vec{F}_{\text {int }}^{* *}\right]^{\xi}=\frac{1}{2} \int_{V_{0}}\left\{\left[\mathbf{I}+\mathbf{f}_{n}^{n+1}\right]\left[\mathbf{\Sigma}^{n+1^{\mathrm{T}}}+\mathbf{C}^{* *}\right] \mathbf{f}_{0}^{n+1^{\mathrm{T}}} \vec{D}^{\xi} J_{0}^{n+1}\right\} \mathrm{d} V_{0},}
\end{aligned}
$$

where tensors $\mathbf{C}^{*}$ and $\mathbf{C}^{* *}$ will be derived to verify the conservation of energy.

Since $\sum_{\xi} \vec{D}^{\xi}=0$, the conservation of the linear momentum $(2.20)$ is directly verified.

Assuming that the Cauchy stress tensors and tensors $\mathbf{C}^{*}$ and $\mathbf{C}^{* *}$ are symmetric, the conservation of the angular momentum (2.22) is obtained from Eq. (2.29) after some algebra [33,34].

For general hypoelastic models, no internal potential exists. Therefore, Eq. (2.24) has to be verified through the evaluation of the work of the internal forces on a loading/unloading cycle. Let $\mathbf{U}_{n}^{\mathbf{e}^{n+1}}$ be the incremental tensor of elastic deformation such that

$$
\mathscr{H}: \frac{1}{2} \ln \left[\mathbf{U}_{n}^{\mathrm{e}^{n+1}} \mathbf{U}_{n}^{\mathrm{e}}{ }_{n}^{n+1}\right]=\mathscr{H}: \mathbf{E}_{n}^{n+1}-\mathbf{s}_{n}^{c n+1} .
$$

Plastic Green-Lagrange tensor $\mathbf{G L}_{n}{ }_{n}^{\mathbf{p}^{n+1}}$, and plastic Almansi tensor $\mathbf{A}_{n}^{\mathbf{p}}{ }_{n}^{n+1}$ are defined from $\mathbf{U}_{n}^{\mathrm{e}{ }^{n+1}}$ as 


$$
\begin{aligned}
& \mathbf{G L}^{\mathbf{p}^{\mathbf{p}^{n+1}}}{ }_{n} \equiv \mathbf{G} \mathbf{L}_{n}^{n+1}-\frac{1}{2}\left[\mathbf{U}_{n}^{\mathbf{e l}^{n+1}} \mathbf{U}_{n}^{\mathrm{e}^{n+1}}-\mathbf{I}\right], \\
& \mathbf{A}_{n}^{\mathbf{p}^{n}{ }_{n}^{n+1}} \equiv \mathbf{A}_{n}^{n+1}-\frac{1}{2} \mathbf{R}_{n}^{n+1}\left[\mathbf{I}-\mathbf{U}_{n}^{\mathrm{e}^{n+1} 1^{-1}} \mathbf{U}_{n}^{\mathrm{e}^{n+1}}{ }_{n}^{-1}\right] \mathbf{R}_{n}^{n+1^{\mathrm{T}}} .
\end{aligned}
$$

In [33], we have proved that Eq. (2.24) could then be rewritten

$$
\Delta_{\text {int }}=\frac{1}{2} \int_{V_{0}}\left\{\left[\mathbf{G L}_{n}^{\mathbf{p l}^{n+1}}: \mathbf{\Sigma}^{n}+\mathbf{G L}_{n}^{n+1}: \mathbf{C}^{*}\right] J_{0}^{n}\right\} \mathrm{d} V_{0}+\frac{1}{2} \int_{V_{0}}\left\{\left[\mathbf{A}_{n}^{\mathbf{p l}^{n+1}}: \mathbf{\Sigma}^{n+1}+\mathbf{A}_{n}^{n+1}: \mathbf{C}^{* *}\right] J_{0}^{n+1}\right\} \mathrm{d} V_{0} .
$$

Therefore, this last relation is the only one that remains to be proved. It can easily be done by setting

$$
\begin{aligned}
\mathbf{C}^{*}= & \frac{\frac{D_{\text {int }}}{J_{0}^{n}}-\mathbf{\Sigma}^{n}: \mathbf{G L}_{n}^{\mathbf{p l}^{n+1}}}{\mathbf{G L}_{n}^{n+1}: \mathbf{G L}_{n}^{n+1}} \mathbf{G L}_{n}^{n+1}, \\
\mathbf{C}^{* *}= & \frac{\frac{D_{\text {int }}}{J_{0}^{n+1}}-\mathbf{\Sigma}^{n+1}: \mathbf{A}_{n}^{\mathbf{p l}^{n+1}}}{\mathbf{A}_{n}^{n+1}: \mathbf{A}_{n}^{n+1}} \mathbf{A}_{n}^{n+1}
\end{aligned}
$$

with

$$
D_{\text {int }} \simeq \frac{1}{2} \varepsilon_{n}^{p n+1}\left[\sum_{v}^{n+1} J_{0}^{n+1}+\sum_{v}^{n} J_{0}^{n}\right],
$$

where $\varepsilon_{n}^{p n+1}=\varepsilon^{p n+1}-\varepsilon^{p n}$ is the equivalent plastic strain increment and $\Sigma_{v}$ the equivalent von Mises yield stress. Thanks to Eqs. (2.32) and (2.33), Eq. (2.24) can then be reduced to

$$
\Delta_{\text {int }}=\int_{V_{0}}\left\{D_{\text {int }}\right\} \mathrm{d} V_{0}>0
$$

Since $D_{\text {int }}$ defined by Eq. (2.34) corresponds to the plastic dissipation by unit of initial volume [34], the Eq. (2.35) is verified and therefore, the time integration scheme is energetically consistent.

\subsubsection{Iterative solution of the algorithm}

In this section, the system of Eqs. (2.16), (2.17) and (2.18) is iteratively resolved by a predictor corrector algorithm. Since this methodology of resolution will be needed to establish our balance method in Section 3 , we present here the principle with some details.

Predicted values (iteration 0 at configuration $n+1$ ) are

$$
\begin{aligned}
{\left[\vec{x}^{n+1,0}\right]^{\xi} } & =\left[\vec{x}^{n}+\Delta t \dot{\vec{x}}^{n}+\frac{\Delta t^{2}}{4} \ddot{\vec{x}}^{n}\right]^{\xi}, \\
{\left[\dot{\vec{x}}^{n+1,0}\right]^{\xi} } & =\left[\dot{\vec{x}}^{n}+\frac{\Delta t}{2} \ddot{\vec{x}}^{n}\right]^{\xi}, \\
{\left[\ddot{\vec{x}}^{n+1,0}\right]^{\xi} } & =0 .
\end{aligned}
$$

These nodal values are compatible with Eqs. (2.16) and (2.17) and are now corrected to satisfy the Eq. (2.18).

Residual $i$ of the Newton-Raphson scheme, at configuration $n+1$, is obtained by

$$
\left[\Delta \vec{F}^{i}\right]^{\xi}=\frac{1}{2} M^{\xi \mu}\left[\ddot{\vec{x}}^{n+1, i}+\ddot{\vec{x}}^{n}\right]^{\mu}+\left[\vec{F}_{\text {int }}^{n+\frac{1}{2}}\left(\vec{x}^{n+1, i}\right)-\vec{F}_{\text {ext }}^{n+\frac{1}{2}}\left(\vec{x}^{n+1, i}\right)\right]^{\xi}
$$

The balance Eq. (2.18) is linearized with respect to the accelerations. Therefore the correction of iteration $i+1$ at configuration $n+1$ is 


$$
\begin{aligned}
& \mathbf{S}^{i \xi^{\xi} \mu}\left[\Delta \ddot{\vec{x}}^{i+1}\right]^{\mu}=-[\Delta \vec{F}]^{i}, \\
& {\left[\ddot{\vec{x}}^{n+1, i+1}\right]^{\mu}=\left[\ddot{\vec{x}}^{n+1, i}+\Delta \ddot{\vec{x}}^{i+1}\right]^{\mu},} \\
& {\left[\dot{\vec{x}}^{n+1, i+1}\right]^{\mu}=\left[\dot{\vec{x}}^{n+1, i}+\frac{\Delta t}{2} \Delta \ddot{x}^{i+1}\right]^{\mu},} \\
& {\left[\vec{x}^{n+1, i+1}\right]^{\mu}=\left[\vec{x}^{n+1, i}+\frac{\Delta t^{2}}{4} \Delta \ddot{\vec{x}}^{i+1}\right]^{\mu},}
\end{aligned}
$$

where $\mathbf{S}$ is the Jacobian matrix defined by

$$
\mathbf{S}^{\xi \mu}=\frac{\partial\left\{\frac{1}{2} M^{\xi v}\left[\ddot{\vec{x}}^{n+1}+\ddot{\vec{x}}^{n}\right]^{v}+\left[\vec{F}_{\mathrm{int}}^{n+\frac{1}{2}}-\vec{F}_{\mathrm{ext}}^{n+\frac{1}{2}}\right]^{\xi}\right\}}{\partial\left[\ddot{\vec{x}}^{n+1}\right]^{\mu}} .
$$

Let $\mathbf{K}^{\xi \mu}$ be the tangent stiffness matrix

$$
\mathbf{K}^{\xi \mu}=\frac{\partial\left[\vec{F}_{\text {int }}^{n+\frac{1}{2}}\right]^{\xi}}{\partial\left[\vec{x}^{n+1}\right]^{\mu}}-\frac{\partial\left[\vec{F}_{\text {ext }}^{n+\frac{1}{2}}\right]^{\xi}}{\partial\left[\vec{x}^{n+1}\right]^{\mu}} .
$$

Therefore, if (2.39) is compared to (2.40), it leads (with I the unity tensor) to

$$
\mathbf{S}^{\xi \mu}=\mathbf{K}^{\xi \nu} \frac{\partial \vec{x}^{v}}{\partial \ddot{\vec{x}}^{\mu}}+\frac{1}{2} M^{\xi \mu} \mathbf{I}
$$

Thanks to Eqs. (2.16) and (2.17), it comes

$$
\frac{\partial \vec{x}^{v}}{\partial \ddot{\vec{x}}^{\mu}}=\frac{\Delta t^{2}}{4} \mathbf{I} \delta_{v \mu}
$$

Finally Eq. (2.41) becomes

$$
\mathbf{S}^{\xi \mu}=\mathbf{K}^{\xi \mu}\left[\frac{\Delta t^{2}}{4} \mathbf{I}\right]+\frac{1}{2} M^{\xi \mu} \mathbf{I} .
$$

After each iteration, Eqs. (2.16) and (2.17) are verified. Moreover, we will admit that Eq. (2.18) is satisfied if the following relative norm of the out-of-equilibrium forces $\Delta \vec{F}$, defined by Eq. (2.37), gets lower than a given tolerance Tol (typically $\mathrm{Tol}=10^{-6}$ ), so that balance equations are satisfied if

$$
\frac{\Delta \vec{F}^{i+1^{\xi}} \cdot \Delta \vec{F}^{i+1^{\xi}}}{\left[\vec{F}_{\text {int }}^{n+\frac{1}{2}}\left(\vec{x}^{n+1}\right)\right]^{\xi} \cdot\left[\vec{F}_{\text {int }}^{n+\frac{1}{2}}\left(\vec{x}^{n+1}\right)\right]^{\xi}+\left[\vec{F}_{\text {ext }}^{n+\frac{1}{2}}\left(\vec{x}^{n+1}\right)\right]^{\xi} \cdot\left[\vec{F}_{\text {ext }}^{n+\frac{1}{2}}\left(\vec{x}^{n+1}\right)\right]^{\xi}}<\text { Tol. }
$$

\section{Balance of the explicit time steps}

The goal of this section is to balance the explicit steps in a thermodynamical consistent way, including the non-linear range. Thanks to this balance, a traditional explicit computation can provide stable initial conditions for a subsequent traditional implicit simulation after an algorithmic switch from explicit to implicit. First, the principle of the balance is presented. Then we will detail the equations in the special case of a spring model and in the special case of an elasto-plastic hypoelastic model. 


\subsection{Principle}

Let us assume to have reached time $t^{n}$ with a traditional explicit central-difference scheme. The explicit steps are of size $\Delta t_{\text {expl }}$. At this time we want to compute the remaining part of the simulation with an implicit Energy Momentum Conserving Algorithm with a time step size $\Delta t_{\text {impl }}$, that is much larger than $\Delta t_{\text {expl }}$. We restrict the first implicit time step size to be a multiple (by an integer $r^{*}$ ) of the explicit time step i.e.

$$
\Delta t_{\mathrm{impl}}=r^{*} \Delta t_{\mathrm{expl}} \text {. }
$$

Factor $r^{*}$ is a user defined parameter or could be evaluated from an integration error [17,19]. Therefore we proceed as described in Fig. 1. The nodal values, and the values at the Gauss points are stored for the configuration $n$. Let us note that we have reached this point with a traditional explicit scheme, but these values will be used as starting values for the balance. Next, $r^{*}$ explicit time steps are computed to reach $t=t^{n+r^{*}}$ (this is the time where the implicit computation will actually start). For this configuration, the new nodal positions are stored and denoted by $\vec{x}_{\text {expl }}^{n+r^{*}}$. These values are the only ones of interest at time $t^{n+r^{*}}$ (i.e. velocities and accelerations computed by the explicit scheme at this time will not be used). They will be used to compute the balanced step.

The equations to be solved for this balance step are the following ones. Let us denote with a subscript impl the values (positions, velocities, accelerations) of the balanced solution at configuration $n+r^{*}$ while the subscript expl denotes the unbalanced solution obtained by the explicit method. Eqs. (2.16) and (2.17) are respectively rewritten as

$$
\left[\vec{x}_{\mathrm{impl}}^{n+r^{*}}\right]^{\xi}=\left[\vec{x}^{n}\right]^{\xi}+\frac{\Delta t_{\mathrm{impl}}}{2}\left[\dot{\vec{x}}_{\mathrm{impl}}^{n+r^{*}}\right]^{\xi}+\frac{\Delta t_{\mathrm{impl}}}{2}\left[\dot{\vec{x}}^{n}\right]^{\xi}
$$

and

$$
\left[\dot{\vec{x}}_{\mathrm{impl}}^{n+r^{*}}\right]^{\xi}=\left[\dot{\vec{x}}^{n}\right]^{\xi}+\frac{\Delta t_{\text {impl }}}{2}\left[\ddot{\vec{x}}_{\mathrm{impl}}^{n+r^{*}}\right]^{\xi}+\frac{\Delta t_{\mathrm{impl}}}{2}\left[\ddot{\vec{x}}^{n}\right]^{\xi},
$$

where the values $\vec{x}_{\text {impl }}^{n+r^{*}}, \dot{\vec{x}}_{\text {impl }}^{n+r^{*}}$ and $\ddot{\vec{x}}_{\text {impl }}^{n+r^{*}}$ are the balanced solutions to be computed at time $t^{n+r^{*}}$. Explicit velocities $\dot{\vec{x}}^{n}$ at time $t^{n}$ can be computed from mid point velocities at time $t^{n-\frac{1}{2}}$ and from acceleration at time $t^{n}$ thanks to Eq. (2.13). The balance Eq. (2.18) is rewritten as

$$
\frac{1}{2} M^{\xi \mu}\left[\ddot{\vec{x}}_{\mathrm{impl}}^{n+r^{*}}+\ddot{\vec{x}}^{n}\right]^{\mu}=\left[\vec{F}_{\mathrm{ext}}^{n+\frac{r^{*}}{2}}-\vec{F}_{\mathrm{int}}^{n+\frac{r^{*}}{2}}\right]^{\xi} \text {. }
$$

The expression of the internal forces $\vec{F}_{\text {int }}^{n+\frac{r^{*}}{2}}$ will be given later.

The system of Eqs. (3.2), (3.3) and (3.4) is solved for $\vec{x}_{\text {impl }}^{n+r^{*}}, \dot{\vec{x}}_{\text {impl }}^{n+r^{*}}$ and $\ddot{\vec{x}}_{\text {impl }}^{n+r^{*}}$ by a predictor corrector algorithm. The predictor values (iteration 0), still verifying Eqs. (3.2) and (3.3), are taken as

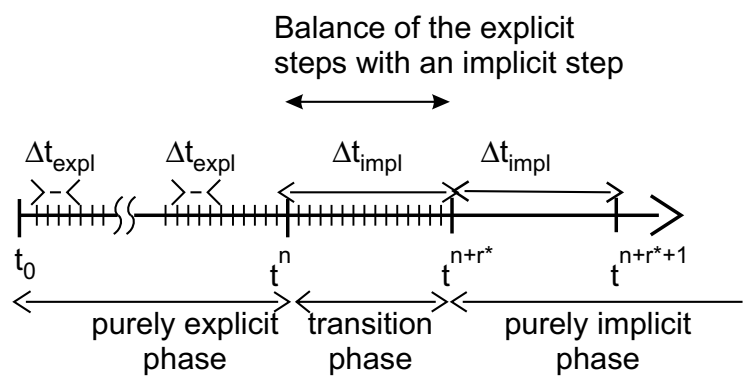

Fig. 1. Description of the method developed to balance the explicit steps when shifting from an explicit simulation to an implicit one. 


$$
\begin{aligned}
& {\left[\vec{x}_{\text {impl }}^{n+r^{*}, 0}\right]^{\xi}=\left[\vec{x}^{n}+\Delta t_{\text {impl }} \dot{\vec{x}}^{n}+\frac{\Delta t_{\text {impl }}^{2}}{4} \ddot{\vec{x}}^{n}\right]^{\xi},} \\
& {\left[\dot{\vec{x}}_{\text {impl }}^{n+r^{*}, 0}\right]^{\xi}=\left[\dot{\vec{x}}^{n}+\frac{\Delta t_{\text {impl }}}{2} \ddot{\vec{x}}^{n}\right]^{\xi},} \\
& {\left[\ddot{\vec{x}}_{\text {impl }}^{n+r^{*}, 0}\right]^{\xi}=0 .}
\end{aligned}
$$

If values predicted by Eq. (3.5) are used to start the correction phase, the balance Eq. (3.4) generally cannot be reached since the values at configuration $n$ (obtained with a traditional explicit scheme) are not balanced, leading to a lack of convergence in the Newton-Raphson scheme. Therefore, $\vec{x}_{\operatorname{expl}}^{n+r^{*}}$ is used as initial predicted positions and the first increment of position is then

$$
\Delta \vec{x}=\vec{x}_{\text {expl }}^{n+r^{*}}-\vec{x}_{\text {impl }}^{n+r^{*}, 0}
$$

and, to be able to still verify Eqs. (3.2) and (3.3), we have a new prediction given by

$$
\begin{aligned}
& {\left[\vec{x}_{\text {impl }}^{n+r^{*}, 1}\right]^{\xi}=\left[\vec{x}_{\text {expl }}^{n+r^{*}}\right]^{\xi},} \\
& {\left[\dot{\vec{x}}_{\text {impl }}^{n+r^{*}, 1}\right]^{\xi}=\left[\dot{\vec{x}}_{\text {impl }}^{n+r^{*}, 0}\right]^{\xi}+\frac{2}{\Delta t_{\mathrm{impl}}}\left[\vec{x}_{\text {expl }}^{n+r^{*}}-\vec{x}_{\text {impl }}^{n+r^{*}, 0}\right]^{\xi},} \\
& {\left[\ddot{\vec{x}}_{\text {impl }}^{n+r^{*}, 1}\right]^{\xi}=\left[\ddot{\vec{x}}_{\mathrm{impl}}^{n+r^{*}, 0}\right]^{\xi}+\frac{4}{\Delta t_{\mathrm{impl}}^{2}}\left[\vec{x}_{\mathrm{expl}}^{n+r^{*}}-\vec{x}_{\mathrm{impl}}^{n+r^{*}, 0}\right]^{\xi} .}
\end{aligned}
$$

From this point, the system is solved iteratively as in Section 2.5.4. Residual (2.37) at iteration $i>1$ for configuration $n+r^{*}$ is rewritten as

$$
\left(\Delta \vec{F}^{i}\right)^{\xi}=M^{\xi \mu}\left[\frac{\ddot{x}_{\text {impl }}^{n+r^{*}, i}+\ddot{\vec{x}}^{n}}{2}\right]^{\mu}+\left[\vec{F}_{\mathrm{int}}^{n+\frac{r^{*}}{2}}\left(\vec{x}_{\mathrm{impl}}^{n+r^{*}, i}\right)-\vec{F}_{\mathrm{ext}}^{n+\frac{r^{*}}{2}}\left(\vec{x}_{\mathrm{impl}}^{n+r^{*}, i}\right)\right]^{\xi}
$$

and the corrections on the nodal values are obtained by

$$
\begin{aligned}
& \mathbf{S}^{i^{\xi} \mu}\left[\Delta \ddot{\vec{x}}^{i+1}\right]^{\mu}=-\left[\Delta \vec{F}^{i}\right]^{\xi}, \\
& {\left[\ddot{\vec{x}}_{\mathrm{impl}}^{n+r^{*}, i+1}\right]^{\mu}=\left[\ddot{\vec{x}}_{\mathrm{impl}}^{n+r^{*}, i}+\Delta \ddot{\vec{x}}^{i+1}\right]^{\mu},} \\
& {\left[\dot{\vec{x}}_{\mathrm{impl}}^{n+r^{*}, i+1}\right]^{\mu}=\left[\dot{\vec{x}}_{\text {impl }}^{n+r^{*}, i}+\frac{\Delta t}{2} \Delta \ddot{x}^{i+1}\right]^{\mu},} \\
& {\left[\vec{x}_{\text {impl }}^{n+r^{*}, i+1}\right]^{\mu}=\left[\vec{x}_{\mathrm{impl}}^{n+r^{*}, i}+\frac{\Delta t^{2}}{4} \Delta \ddot{\vec{x}}^{i+1}\right]^{\mu},}
\end{aligned}
$$

where $\mathbf{S}$ is the tangent matrix obtained in the same way than Eq. (2.39). Iterations are stopped when the balance equation is verified within a given tolerance Tol (see Eq. (2.44)).

In summary, the proposed method is the following:

- from initial conditions at time $t_{0}$, a traditional explicit simulation is led until time $t^{n}$;

- at time $t^{n}$ nodal values and values at Gauss points are stored;

- $r^{*}$ explicit steps are taken from time $t^{n}$ to time $t^{n+r^{*}}$, leading to the displacements $\vec{x}_{\text {expl }}^{n+r^{*}}$;

- displacement $\vec{x}_{\text {expl }}^{n+r^{*}}$ are used, through a predictor-corrector algorithm, to initiate a balance step between time $t^{n}$ (for which values at nodes and Gauss points were stored) and time $t^{n+r^{*}}$;

- once the Newton-Raphson scheme of this balance step has converged, new values obtained at time $t^{n+r^{*}}$ are used as initial conditions for the following implicit time integration. 
Let us note some remarks:

- the described procedure remains valid if we have shifts from an implicit method to an explicit one and more than once shift from an explicit scheme to an implicit one;

- this procedure can be extended to other explicit schemes, such as the $\alpha$-generalized explicit scheme [4] and to an implicit consistent algorithm with numerical dissipation such as the Energy-Dissipative-Conserving-Momentum [39,40].

Now we are able to design the internal force expressions $\vec{F}_{\text {int }}^{n+\frac{r^{*}}{2}}\left(\vec{x}_{\text {impl }}^{n+r^{*}}\right)$ that will lead to a thermodynamical consistent balanced step. To achieve this goal, we have to rewrite the conservation laws for this balanced step.

\subsection{Construction of the internal forces for the balance step}

Since Eqs. (3.2), (3.3) and (3.4) are already satisfied, the conservation laws established in Section 2.5.2, can easily be adapted to obtain the new conditions on $\vec{F}_{\text {int }}^{n+\frac{r^{*}}{2}}\left(\vec{x}_{\text {impl }}^{n+r^{*}}\right)$. These conditions correspond to the physical principles of motion (the internal forces cannot modify the motion of the centre of gravity and the variation of the energy of a system correspond to the work done by the external forces).

Conservation of the linear momentum (2.19) is rewritten

$$
\vec{L}^{n+r^{*}}-\vec{L}^{n}=\Delta t \sum_{\xi}\left[\vec{F}_{\mathrm{ext}}^{n+r^{*}}\right]^{\xi}
$$

and is verified if we have

$$
\sum_{\xi}\left[\vec{F}_{\text {int }}^{n+\frac{r^{*}}{2}}\right]^{\xi}=0 .
$$

Conservation of the angular momentum (2.21) is rewritten as

$$
\vec{J}^{n+r^{*}}-\vec{J}^{n}=\frac{\Delta t}{2}\left[\vec{x}_{\mathrm{impl}}^{n+r^{*}}+\vec{x}^{n}\right]^{\xi} \wedge\left[\vec{F}_{\mathrm{ext}}^{n+\frac{r^{*}}{2}}\right]^{\xi},
$$

which is verified if the internal forces satisfy

$$
\left[\frac{\vec{x}_{\mathrm{impl}}^{n+r^{*}}+\vec{x}^{n}}{2}\right]^{\xi} \wedge\left[\vec{F}_{\mathrm{int}}^{n+r^{*}}\right]^{\xi}=0 .
$$

Conservation of the energy (2.23) is rewritten as

$$
E^{n+r^{*}}-E^{n}=W_{\mathrm{ext}}^{n+r^{*}}-W_{\mathrm{ext}}^{n}-\Delta_{\mathrm{int}}
$$

that leads to the following condition

$$
\left[\vec{F}_{\text {int }}^{n+\frac{r^{*}}{2}}\right]^{\xi} \cdot\left[\vec{x}^{n+r^{*}}-\vec{x}^{n}\right]^{\xi}=U_{\text {int }}^{n+r^{*}}-U_{\text {int }}^{n}+\Delta_{\text {int }} .
$$

Therefore, the internal forces $\vec{F}_{\text {int }}^{n+\frac{r^{*}}{2}}\left(\vec{x}_{\text {impl }}^{n+r^{*}}\right)$ have to be constructed so that they verify Eqs. (3.11), (3.13) and (3.15). By similitude with Section 2.5.3, the expression of the internal forces (2.25) and (2.29) (for respectively the spring and the bulk element) are adapted by computing these expressions between configurations $n$ and $n+r^{*}$ and not between configurations $n$ and $n+1$. Therefore, we just give the final results. 
Expression of the balanced internal forces for a spring model (2.25) is rewritten as

$$
\begin{aligned}
{\left[\vec{F}_{\mathrm{int}}^{n+\frac{r^{*}}{2}}\right]^{\xi}=} & \frac{U\left(l^{n+r^{*}}\right)-U\left(l^{n}\right)}{l^{n+r^{* 2}}-l^{n^{2}}}\left\{\left[\vec{x}^{n+r^{*}}+\vec{x}^{n}\right]^{\xi}-\left[\vec{x}^{n+r^{*}}+\vec{x}^{n}\right]^{\mu}\right\} \quad \text { if } l^{n+r^{*}} \neq l^{n} \\
= & \frac{\frac{\partial}{\partial l} U\left(\frac{l^{n+r^{*}}+l^{n}}{2}\right)}{l^{n+r^{*}}+l^{n}}\left\{\left[\vec{x}^{n+r^{*}}+\vec{x}^{n}\right]^{\xi}-\left[\vec{x}^{n+r^{*}}+\vec{x}^{n}\right]^{\mu}\right\} \quad \text { if } l^{n+r^{*}} \rightarrow l^{n}
\end{aligned}
$$

that satisfies Eqs. (3.11), (3.13) and (3.15).

Expression of the balanced internal forces for a hypoelastic model (2.29) is rewritten as

$$
\begin{aligned}
& {\left[\vec{F}_{\text {int }}^{n+\frac{r^{*}}{2}}\right]^{\xi}=\frac{1}{2}\left[\vec{F}_{\text {int }}^{*}+\vec{F}_{\text {int }}^{* *}\right]^{\xi},} \\
& {\left[\vec{F}_{\text {int }}^{*}\right]^{\xi}=\frac{1}{2} \int_{V_{0}}\left\{\left[\mathbf{I}+\mathbf{F}_{n}^{n+r^{*}}\right]\left[\mathbf{\Sigma}^{n \mathrm{~T}}+\mathbf{C}^{*}\right] \mathbf{f}_{0}^{n \mathrm{~T}} \vec{D}^{\xi} J_{0}^{n}\right\} \mathrm{d} V_{0},} \\
& {\left[\vec{F}_{\text {int }}^{* *}\right]^{\xi}=\frac{1}{2} \int_{V_{0}}\left\{\left[\mathbf{I}+\mathbf{f}_{n}^{n+r^{*}}\right]\left[\mathbf{\Sigma}^{n+r^{* \mathrm{~T}}}+\mathbf{C}^{* *}\right] \mathbf{f}_{0}^{n+r^{* \mathrm{~T}}} \vec{D}^{\xi} J_{0}^{n+r^{*}}\right\} \mathrm{d} V_{0},}
\end{aligned}
$$

where $\mathbf{C}^{*}$ and $\mathbf{C}^{* *}$ will be defined. In this last relation, we use the deformation gradient $\mathbf{F}$ between the configurations $n$ and $n+r^{*}$

$$
\mathbf{F}_{n}^{n+r^{*}}=\frac{\partial \vec{x}_{\text {impl }}^{n+r^{*}}}{\partial \vec{x}^{n}}
$$

and Cauchy stress tensor obtained by

$$
\boldsymbol{\Sigma}^{n+r^{*}}=\mathbf{R}_{n}^{n+r^{*}}\left[\mathbf{\Sigma}^{n}+\mathscr{H}: \mathbf{E}_{n}^{n+r^{*}}-\mathbf{s}^{\mathbf{c}}\right] \mathbf{R}_{n}^{n+r^{*} \mathrm{~T}}
$$

with $\mathbf{E}_{n}^{n+r^{*}}$ and $\mathbf{R}_{n}^{n+r^{*}}$ computed from $\mathbf{F}_{n}^{n+r^{*}}$, and with $\mathbf{s}^{\mathbf{c}}$ the viscoplastic corrections obtained by the von Mises criterion evaluated at time $t^{n+r^{*}}$. Using $\mathbf{F}_{n}^{n+r^{*}}$ to compute strain tensors, the two tensors $\mathbf{C}^{*}$ and $\mathbf{C}^{* *}$ are defined by

$$
\begin{aligned}
\mathbf{C}^{*}= & \frac{\frac{D_{\text {int }}}{J_{0}^{n}}-\boldsymbol{\Sigma}^{n}: \mathbf{G L}_{n}^{\mathbf{p l}^{n+r^{*}}}}{\mathbf{G L}_{n}^{n+r^{*}}: \mathbf{G L}_{n}^{n+r^{*}}} \mathbf{G L}_{n}^{n+r^{*}}, \\
\mathbf{C}^{* *}= & \frac{\frac{D_{\text {int }}}{J_{0}^{n+r^{*}}}-\boldsymbol{\Sigma}^{n+r^{*}}: \mathbf{A}_{n}^{\mathbf{p} \mathbf{l}^{n+r^{*}}}}{\mathbf{A}_{n}^{n+r^{*}}: \mathbf{A}_{n}^{n+r^{*}}} \mathbf{A}_{n}^{n+r^{*}}
\end{aligned}
$$

with

$$
D_{\mathrm{int}} \simeq \frac{1}{2} \varepsilon_{n}^{p n+r^{*}}\left[\sum_{v}^{n+r^{*}} J_{0}^{n+r^{*}}+\sum_{v}^{n} J_{0}^{n}\right]
$$

the internal dissipation between time $t^{n}$ and time $t^{n+1}$.

Similitudes in the construction of the internal forces with Section 2.5.3, allow us to claim (this is rigorously demonstrated in Appendix A) that Eq. (3.17) satisfies Eqs. (3.11), (3.13) and (3.15) and therefore, the time step between $t^{n}$ and $t^{n+r^{*}}$ is thermodynamically consistent.

\section{Numerical examples}

In this section, we will prove numerically that our method of transition between an explicit time integration and an implicit time integration does not lead to numerical instability in the non-linear range. In a first 
example, we will compute a long-duration non-linear explicit simulation with the balance method. It will allow us to prove that the proposed methodology ensures stability in the non-linear range. Nevertheless the goal of this paper is not to balance a whole explicit simulation, but to use this methodology to be able to shift from an explicit algorithm to an implicit algorithm. Therefore, in the next two examples, we will use the proposed balance method only when shifting from an explicit simulation to an implicit one. We will then prove that this methodology allows us to combine the two methods without loss of accuracy.

\subsection{Numerical example 1: Dynamics of a mass-spring system}

This example was initially proposed by Armero and Romero [39,40]. It consists of a mass $(m=2 \mathrm{~kg})$ attached to the extremity of a spring (stiffness $k=15 \mathrm{~N} / \mathrm{m}$, length at rest $l_{0}=10 \mathrm{~m}$ ). The other extremity of the spring is fixed. The mass has an initial velocity of $10 \mathrm{~m} / \mathrm{s}$ perpendicular to the spring that is initially unstressed (Fig. 2). Due to the large rotation, the system is geometrically non-linear. Let us point out that the problem is solved in the Cartesian coordinates and not using polar coordinates. We compare the results obtained by the three following methods:

- simulation with an implicit algorithm (EMCA) and a time step of $1.5 \mathrm{~s}$;

- simulation with an explicit algorithm (central difference) and a security factor $\gamma_{s}=0.68465$ that leads to a time step of $\Delta t \simeq 0.5 \mathrm{~s}$ (time step size of the non-linear two-degree of freedom problem is computed by a power iteration method [5] and is therefore not exactly constant $\Delta t \in[0.49 ; 0.51])$. It should be noted that in such a simple case, using a constant time step such as $\Delta t=0.5 \mathrm{~s}$ would lead to a less oscillatory solution. Nevertheless, for more complex situations such as those encountered in industrial applications, one has to use a non-constant time step. Thus, as the purpose of this example is just to illustrate the methodology, we have preferred to use the non-constant time step computed by the power-iteration method;

- simulation with this explicit algorithm, but with each three explicit steps balanced by the proposed method $\left(\Delta t_{\text {impl }}=3 \Delta t_{\text {expl }}\right)$. Note that the simultaneous use of explicit and implicit schemes in this example is purely for purposes of illustrating the properties of the proposed balancing algorithm. The actual use of balancing when shifting from explicit to implicit will be illustrated by the next examples.

Fig. 3 represents the time evolution of the spring length and Fig. 4 represents the time evolution of the energies. The implicit solution (Figs. 3(a) and 4(a)) gives a consistent solution where the oscillations (in the length, the kinematic energy and the potential energy) have the amplitudes obtained by other authors $[39,40]$. The explicit solution (Figs. 3(b) and 4(b)) is unconsistent since the total energy is not constant and since the oscillations do not have a constant amplitude. This comes from the non-linearities that are approximatively integrated with the explicit scheme (these oscillations are also reinforced by the use of a

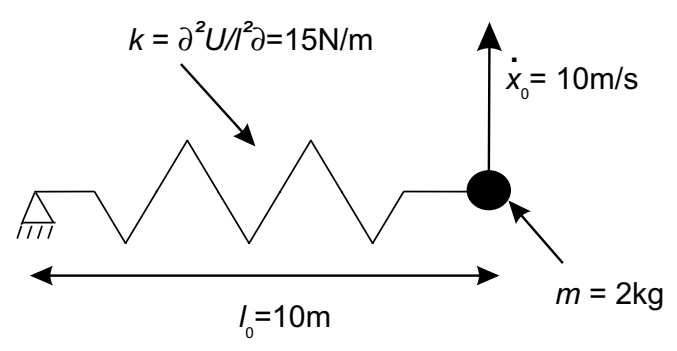

Fig. 2. Sketch of the mass-spring system. 

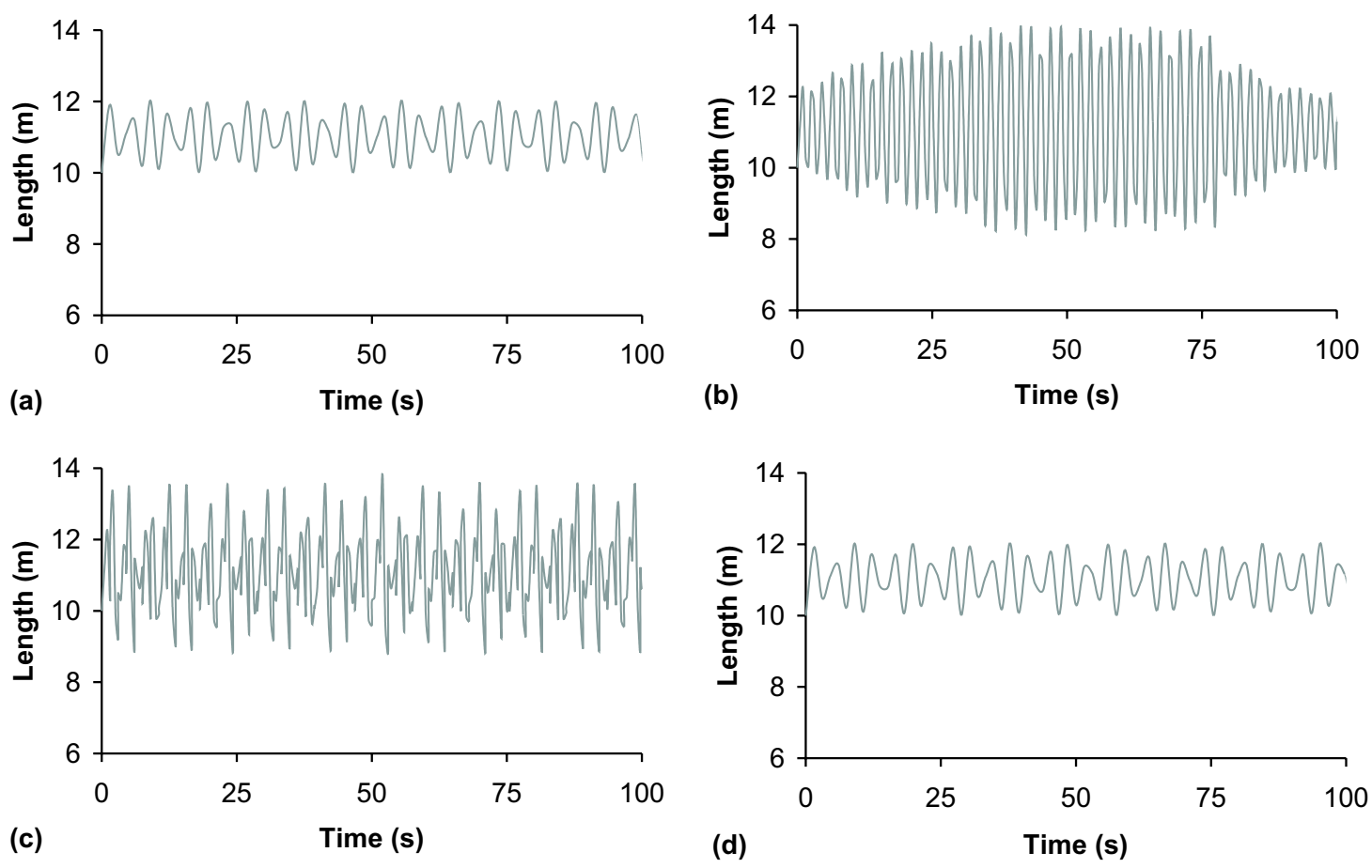

Fig. 3. Time evolution of the spring length. (a) Implicit resolution with EMCA, (b) explicit resolution (central difference), (c) explicit resolution balanced by an implicit algorithm (balanced and unbalanced times are plotted), (d) explicit resolution balanced by an implicit algorithm (only balanced times are plotted).

non-strictly-constant time step). Now we balance the explicit steps with the developed method (each three explicit steps are balanced). Figs. 3(c) and 4(c) illustrate, respectively the length and the energies for each explicit step (balanced and unbalanced). Contrarily to the explicit solution, even if the total energy is not constant, the oscillations are limited. In fact, the unbalanced explicit steps, that are unstable (we are under the stability limit that is established only for linear systems, and no longer remains necessarily true in this non-linear example) lead to an increase in the total energy. But the balance method provides consistent results after three explicit steps. If only the balanced solutions are represented (Figs. 3(d) and 4(d)), the solution obtained is consistent and similar to the implicit solution. Finally, let us note that the implicit solution requires the resolution of 749 Newton-Raphson iterations and that the balanced explicit solution requires the resolution of 671 Newton-Raphson iterations.

Let us also point out that if the standard explicit simulation leads to unbounded oscillations in the total energy, which results from the fact that time step size is non-constant (time step size of the two-degree of freedom problem is computed by the power iteration method [5] with $\Delta t \in[0.49 ; 0.51])$. The computed step size is always lower than the critical one, but its variation leads to the presented results. If we use a constant time step, the oscillations in the energy are bounded. For example we illustrate the length evolution (Fig. 5) and the energy evolution (Fig. 6) for two constant time step sizes ( $\Delta t=0.05 \mathrm{~s}$ and $0.5 \mathrm{~s}$ ). Since the explicit scheme with constant time step is symplectic, the total energy is bounded. Amplitude of the oscillations increases with the time step size. Nevertheless small variations in the time step size lead to instability. For more complex systems than a mass/spring one, these variations in the step size cannot be avoided.

This example allows us to conclude that the balance of explicit steps is stable and consistent in the nonlinear range and does not require too many iterations. Therefore, we can use it without destabilizing the 

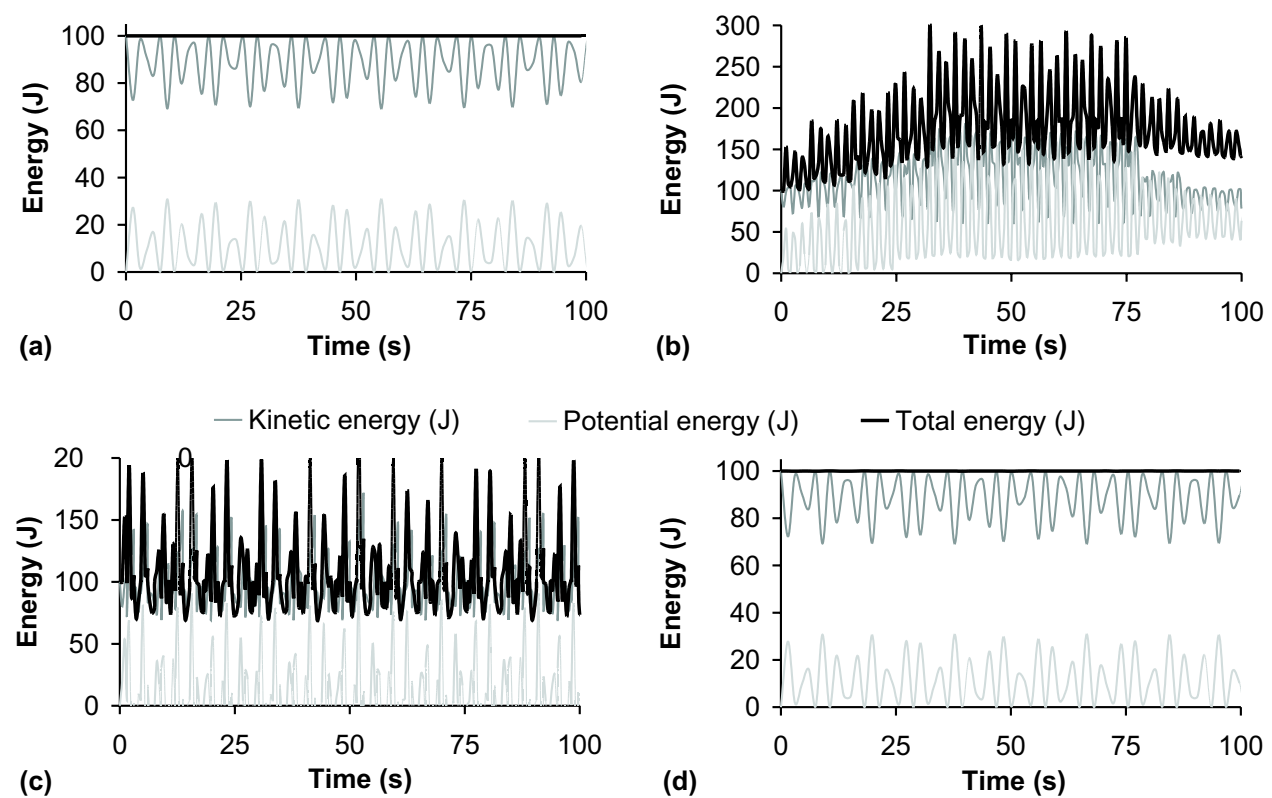

Fig. 4. Time evolution of the mass-spring system energy. (a) Implicit resolution with EMCA, (b) explicit resolution (central difference), (c) explicit resolution balanced by an implicit algorithm (balanced and unbalanced times are plotted), (d) explicit resolution balanced by an implicit algorithm (only balanced times are plotted).

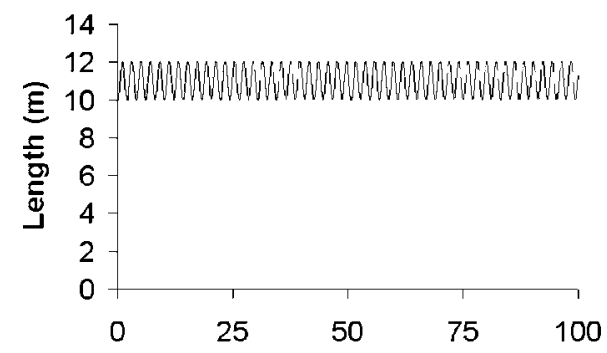

(a)

Time (s)

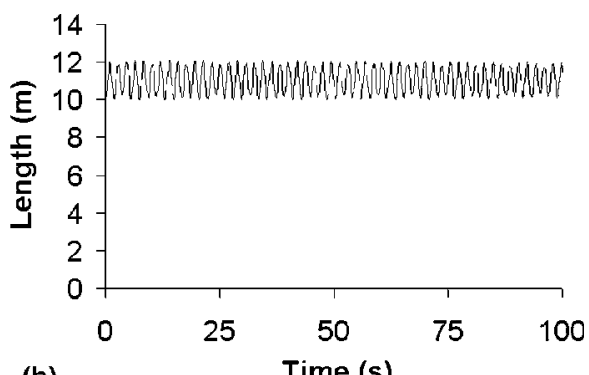

(b)

Fig. 5. Time evolution of the spring length with an explicit scheme and a constant time step size. (a) $\Delta t=0.05 \mathrm{~s}$, (b) $\Delta t=0.5 \mathrm{~s}$.

computation. In this example, the balanced solution was used to stabilize a fully explicit simulation. But we have developed it to be used only when shifting from an explicit scheme to an implicit one. Therefore, we will study academic examples where we will shift from an integration method to another one. When shifting from an explicit method to an implicit one, the proposed method will be used.

\subsection{Numerical example 2: Dynamics of a $3 D$ beam}

The dynamics of a cantilever beam is studied. The beam is discretized into 320 (4 in width, 4 in height and 20 in length) elements. Its geometry is illustrated in Fig. 7. Dimensions and material properties are 

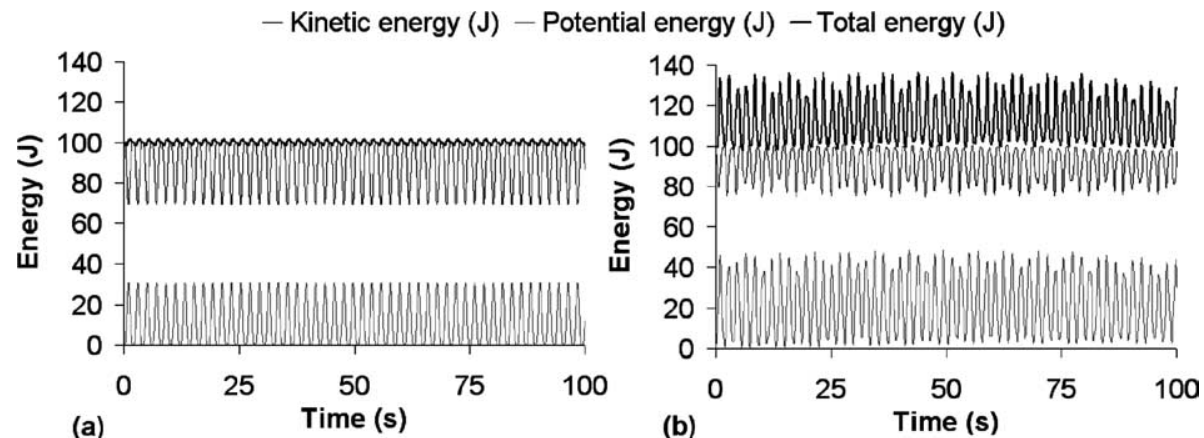

Fig. 6. Time evolution of the mass-spring system energy with an explicit scheme and a constant time step size. (a) $\Delta t=0.05 \mathrm{~s}$, (b) $\Delta t=0.5 \mathrm{~s}$.

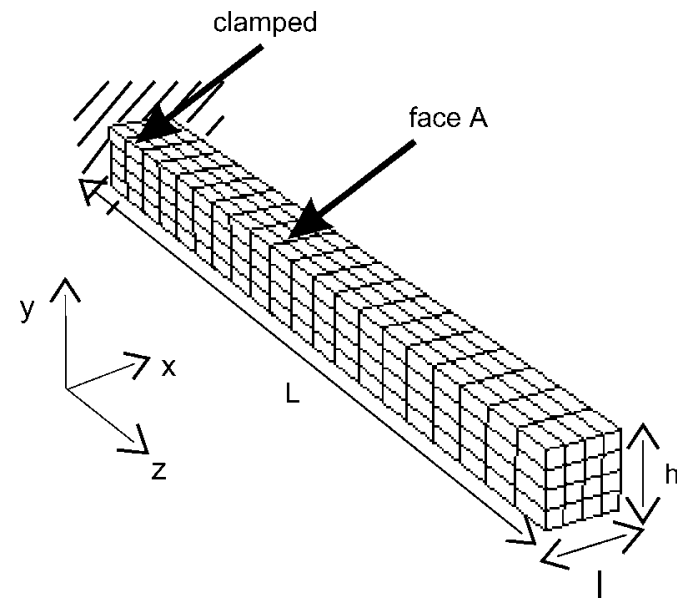

Fig. 7. Initial geometry and mesh for the beam problem.

Table 1

Geometrical and material properties of the beam

\begin{tabular}{ll}
\hline Property & Value \\
\hline Length & $L=100 \mathrm{~mm}$ \\
Width & $l=8 \mathrm{~mm}$ \\
Height & $h=8 \mathrm{~mm}$ \\
Density & $\rho=10,000 \mathrm{~kg} / \mathrm{m}^{3}$ \\
Young's modulus & $Y=186,000 \mathrm{~N} / \mathrm{mm}^{2}$ \\
Poisson's ratio & $v=0.3$ \\
Yield stress & $\Sigma_{v}=20,000+16,000 \varepsilon^{p} \mathrm{~N} / \mathrm{mm}^{2}$ \\
\hline
\end{tabular}

reported in Table 1. On top of face A (Fig. 7), a time dependent force is applied at each node. This force is computed from 


$$
\left(\begin{array}{c}
\vec{F}_{x} \\
\vec{F}_{y} \\
\vec{F}_{z}
\end{array}\right)=\left(\begin{array}{c}
0 \\
350 \\
0
\end{array}\right) \mathrm{N} / \mathrm{ms} \times\left\{\begin{array}{cc}
t, & 0 \leqslant t \leqslant 3 \mathrm{~ms} \\
(6 \mathrm{~ms}-t), & 3 \mathrm{~ms}<t \leqslant 6 \mathrm{~ms}
\end{array}\right\} .
$$

After $6 \mathrm{~ms}$, the forces are relaxed. We compare the solutions obtained for all times between 0 and $10 \mathrm{~ms}$ by:

- an implicit simulation (EMCA) with a time step equal to $0.01 \mathrm{~ms}$,

- an explicit simulation (central difference) with a security factor $\gamma_{s}=0.626764$, that leads to a time step size $\Delta t=0.25 \mu \mathrm{s}$,

- an implicit/explicit combined algorithm that uses the implicit scheme (with $\Delta t_{\text {impl }}=0.01 \mathrm{~ms}$ ) between $t=0 \mathrm{~ms}$ and $t=2 \mathrm{~ms}$ and between $t=3 \mathrm{~ms}$ and $t=10 \mathrm{~ms}$. The explicit scheme (central difference) is used when the loading reaches its maximal value between $t=2 \mathrm{~ms}$ and $t=3 \mathrm{~ms}$. The balance of the explicit steps is then activated, with $\Delta t_{\mathrm{impl}}=40 \Delta t_{\mathrm{expl}}$, between time $t=3 \mathrm{~ms}$ and time $t=3.01 \mathrm{~ms}$.

Fig. 8(a) illustrates the time evolution of the plastically dissipated energy. All the computed solutions are similar (maximum difference of $0.3 \%$ ). For the combined method, no perturbation appear when the algorithm shifts from an implicit method to an explicit method at time $t=2 \mathrm{~ms}$ or when the explicit algorithm shifts back to an implicit method at time $t=3 \mathrm{~ms}$. The same observations are obtained by analyzing the vertical displacement of the extremity of the beam (Fig. 8(b)). During the loading $(t<3 \mathrm{~ms})$, the unloading ( $3 \mathrm{~ms} \leqslant t<6 \mathrm{~ms})$, and the free vibration of the beam $(t \leqslant 10 \mathrm{~ms})$, the solutions are identical in amplitude and in phase. The equivalent plastic strain obtained after the loading (Fig. 9(a)) and after $10 \mathrm{~ms}$ (Fig. 9(b)) are also identical.

\subsection{Numerical example 3: Taylor's bar problem}

The impact of a cylindrical bar, with an initial velocity $\dot{\vec{x}}_{0}$ is studied. Due to the symmetry, only a fourth of the cylinder is discretized into 576 elements (48 in the base and 12 in length). The initial geometry is illustrated in Fig. 10. Dimensions and material properties are reported in Table 2. The total simulation time is $80 \mu \mathrm{s}$. We compare the solutions obtained by:

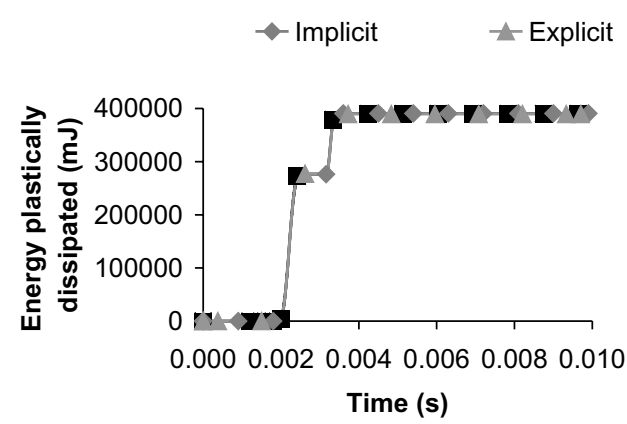

(a)

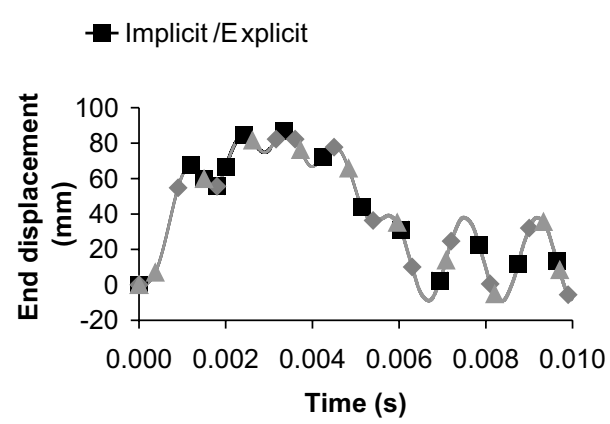

(b)

Fig. 8. Time evolution of the results for the beam. (a) Energy plastically dissipated, (b) displacement (along $y$ ) of the extremity. 

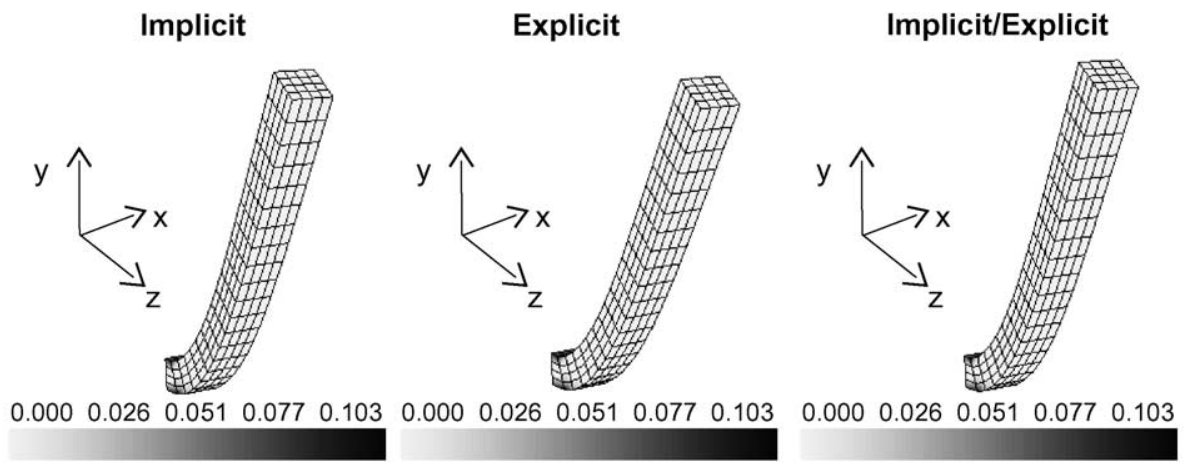

(a)
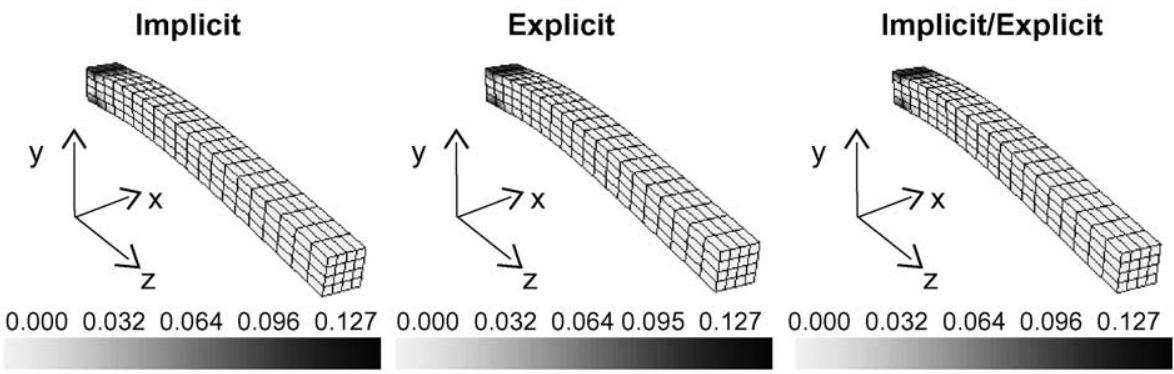

(b)

Fig. 9. Equivalent plastic strain for the beam after (a) $t=3 \mathrm{~ms}$, (b) $t=10 \mathrm{~ms}$.

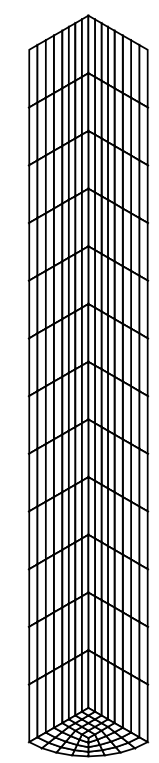

Fig. 10. Initial geometry and mesh for Taylor's bar. 
Table 2

Geometrical and material properties of Taylor's bar

\begin{tabular}{ll}
\hline Property & Value \\
\hline Length & $L=32.4 \mathrm{~mm}$ \\
Radius & $r=3.2 \mathrm{~mm}$ \\
Initial velocity & $\dot{\vec{x}_{0}}=227 \mathrm{~m} / \mathrm{s}$ \\
Density & $\rho=8900 \mathrm{~kg} / \mathrm{m}^{3}$ \\
Young's modulus & $Y=117,000 \mathrm{~N} / \mathrm{mm}^{2}$ \\
Poisson's ratio & $v=0.35$ \\
Yield stress & $\Sigma_{v}=400+100 \varepsilon^{p} \mathrm{~N} / \mathrm{mm}^{2}$ \\
\hline
\end{tabular}

- an implicit simulation (EMCA) with a time step equal to $500 \mathrm{~ns}$ (160 steps for the total simulation),

- an explicit simulation (central difference) with a security factor $\gamma_{s}=0.8$, that leads to an initial time step size $\Delta t=61.3 \mathrm{~ns}$ (1409 steps for the total simulation),

- an implicit/explicit combined algorithm that uses an explicit scheme $\left(\gamma_{s}=0.8\right)$ for $t<40 \mu \mathrm{s}$ and an implicit scheme (with $\Delta t_{\text {impl }}=0.5 \mu \mathrm{s}$ ) for $t>40 \mu \mathrm{s}$. The balance of the explicit steps is reached, with $\Delta t_{\mathrm{impl}}=9 \Delta t_{\mathrm{expl}}$, between time $t=40 \mu \mathrm{s}$ and time $t=40.5 \mu \mathrm{s}$.

Fig. 11(a) illustrates the time evolution of the plastically dissipated energy. All the computed solutions are similar (maximum difference of 1.5\%). The same observations are obtained by analyzing the reversible energy stored (kinetic energy added to the work of the internal forces minus the plastically dissipated energy) in the cylinder (Fig. 11(b)). Since the implicit algorithm is energetically conservative, and since the explicit algorithm uses small time steps, the reversible energy almost corresponds to the initial energy minus the plastically dissipated energy. It remains true for the combined method, demonstrating once again that the shift method, at time $t=40 \mu \mathrm{s}$, is energetically consistent. Nevertheless, by analyzing the final values of the simulation (Table 3), it appears that the energy plastically dissipated with the explicit scheme or with the combined scheme is larger than the initial kinetic energy (equal to $56.27 \mathrm{~J}$ ). This results from the fact that the explicit scheme is not thermodynamically consistent in the non-linear range and not from a lack of stability in the shift method. The final length and radius (of the base) of the cylinder are equivalent by $0.5 \%$ for all methods (Table 3 ). The equivalent plastic strains obtained after the impact (Fig. 12) are also almost identical (the explicit scheme leads to about $7 \%$ higher plastic strain, corresponding to the fact that the plastically dissipated energy is overestimated).

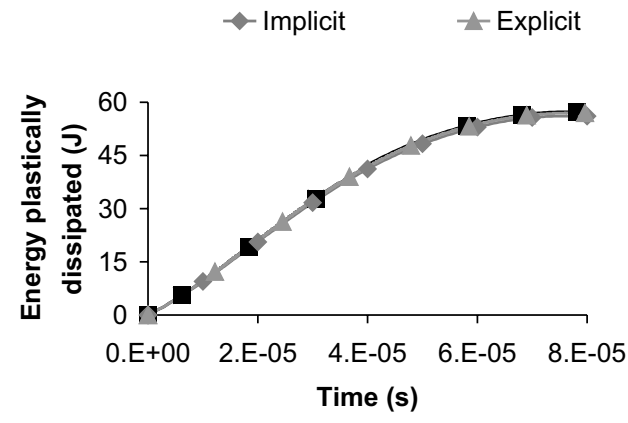

(a)

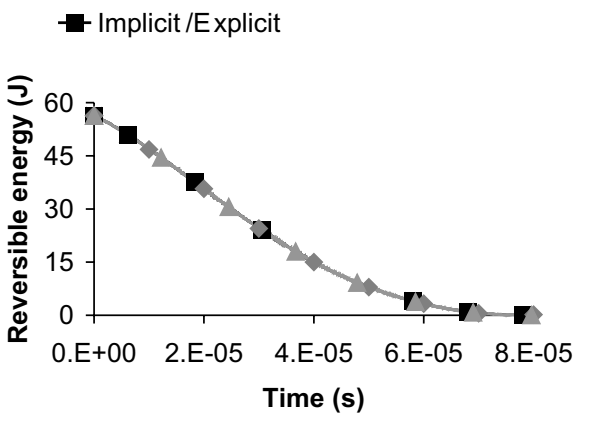

(b)

Fig. 11. Time evolution of some results for Taylor's bar. (a) Plastically dissipated energy, (b) reversible energy. 
Table 3

Comparison of the final results of Taylor's bar

\begin{tabular}{llll}
\hline Values & Full implicit & Full explicit & Combined implicit/explicit \\
\hline Plastically dissipated energy & $\Delta_{\mathrm{int}}=56.25 \mathrm{~J}$ & $\Delta_{\mathrm{int}}=56.97 \mathrm{~J}$ & $\Delta_{\mathrm{int}}=57.33 \mathrm{~J}$ \\
Maximal plastic strain & $\varepsilon_{\max }^{\mathrm{pl}}=2.37$ & $\varepsilon_{\max }^{\mathrm{pl}}=2.54$ & $\varepsilon_{\max }^{\mathrm{pl}}=2.53$ \\
Length & $L=21.5784 \mathrm{~mm}$ & $L=21.6279 \mathrm{~mm}$ & $L=21.5971 \mathrm{~mm}$ \\
Radius & $r=6.8530 \mathrm{~mm}$ & $r=6.8368 \mathrm{~mm}$ & $r=6.8325 \mathrm{~mm}$ \\
\hline
\end{tabular}
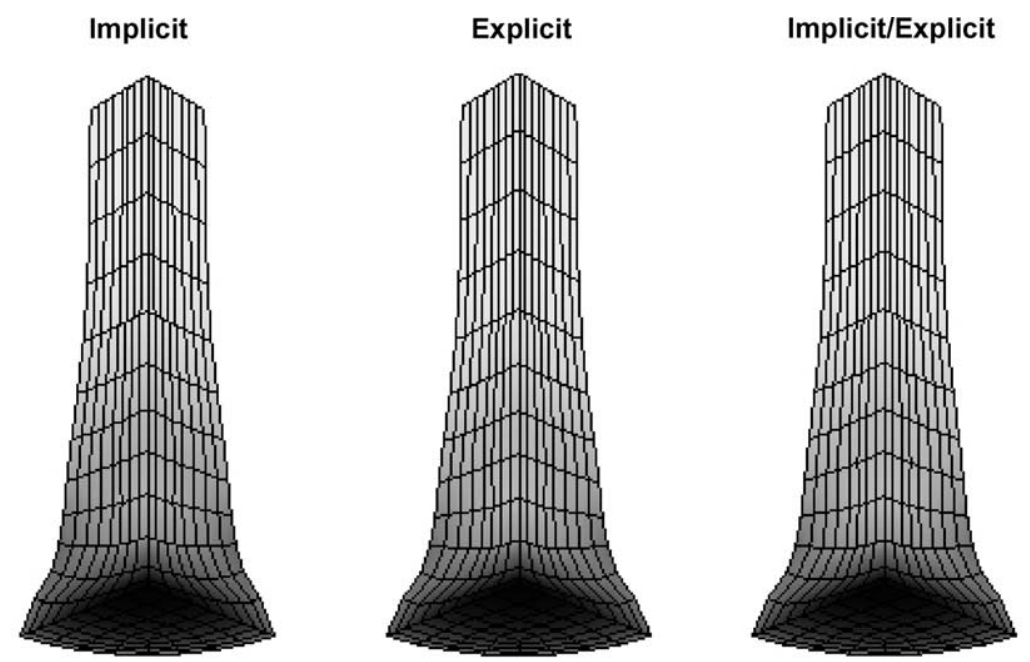

$\begin{array}{llllllllll}0.000 & 0.593 & 1.190 & 1.780 & 2.370 & 0.000 & 0.634 & 1.270 & 1.900 & 2.540\end{array}$

$0.000 \quad 0.6301 .2601 .890 \quad 2.530$

Fig. 12. Equivalent plastic strain for Taylor's bar after $80 \mu \mathrm{s}$.

\section{Conclusions}

In this work, we have proposed a stable and thermodynamically consistent way, even in the non-linear range, to balance some explicit time steps. This method is based on a predictor-corrector algorithm. We have used this methodology for a long-duration simulation of a non-linear mass-spring system to numerically demonstrate its stability in the non-linear range. Next, we have used it to be able to shift from an explicit simulation to an implicit simulation occurring with a larger time step size. The method has been shown to be stable and accurate, and to allow this transition between the two methods to occur without divergence of the iterative procedure.

\section{Appendix A. Consistency of the balance step for the hypoelastic bulk model}

Eq. (3.17) corresponds to the internal force formulation developed to balance the $r^{*}$ last explicit steps. In this section, we will prove that this relation leads to a balanced step that verify the conservation of the linear momentum, the conservation of the angular momentum and the conservation of the energy. 


\section{A.1. Conservation of the linear momentum}

Eq. (3.17) satisfies conservation of the linear momentum defined by Eq. (3.11). It is directly proved by using the property of the shape functions $\sum_{\xi} \vec{D}^{\xi}=0$.

\section{A.2. Conservation of the angular momentum}

Using the fact that the Cauchy stress tensor and tensors $\mathbf{C}^{*}$ and $\mathbf{C}^{* *}$ are symmetric, Eq. (3.17) satisfies, after some algebra, conservation of the angular momentum defined by Eq. (3.13).

\section{A.3. Conservation of the energy}

We have proved in [33] that Eq. (2.24) could then be rewritten as (2.32). Using definitions of Eq. (3.17), the same demonstration reduces Eq. (3.15) to

$$
\begin{aligned}
\Delta_{\mathrm{int}}= & \frac{1}{2} \int_{V_{0}}\left\{\left[\mathbf{G L}_{n}^{\mathbf{p}^{n}{ }_{n}^{n+r^{*}}}: \mathbf{\Sigma}^{n}+\mathbf{G L}_{n}^{n+r^{*}}: \mathbf{C}^{*}\right] J_{0}^{n}\right\} \mathrm{d} V_{0} \\
& +\frac{1}{2} \int_{V_{0}}\left\{\left[\mathbf{A}_{n}^{\mathbf{p}^{n+r^{*}}}: \mathbf{\Sigma}^{n+r^{*}}+\mathbf{A}_{n}^{n+r^{*}}: \mathbf{C}^{* *}\right] J_{0}^{n+r^{*}}\right\} \mathrm{d} V_{0}
\end{aligned}
$$

that becomes the relation to be proved. Thanks to Eqs. (3.20) and (3.21), Eq. (A.1) is reduced to

$$
\Delta_{\text {int }}=\int_{V_{0}}\left\{D_{\text {int }}\right\} \mathrm{d} V_{0}>0 .
$$

Since $D_{\text {int }}$ defined by Eq. (3.21) corresponds to the plastic dissipation by unit of initial volume [34], Eq. (A.2) is satisfied and therefore, the time integration of the balanced step is energetically consistent.

\section{References}

[1] T. Belytschko, T. Hughes, Computational Methods for Transient Analysis, North Holland, 1983.

[2] T. Hughes, The Finite Element Method, Prentice Hall, 1987.

[3] M. Géradin, D. Rixen, Mechanical vibrationsTheory and Applications to Structural Dynamics, John Wiley \& Sons, Paris, 1994.

[4] G. Hulbert, J. Chung, Explicit time integration algorithms for structural dynamics with optimal numerical dissipation, Comput. Methods Appl. Mech. Engrg. 137 (1996) 175-188.

[5] D.J. Benson, Stable time step estimation for multi-material Eulerian hydrocodes, Comput. Methods Appl. Mech. Engrg. 167 (1998) 191-205.

[6] N. Newmark, A method of computation for structural dynamics, J. Eng. Mech. Div. ASCE 85 (EM3) (1959) 67-94.

[7] J. Chung, G. Hulbert, A time integration algorithms for structural dynamics with improved numerical dissipations: The generalized- $\alpha$ method, J. Appl. Mech. 60 (1993) 371-375.

[8] M. Géradin, A. Cardona, Flexible multibody dynamics, A Finite Element Approach, John Wiley \& Sons, 2000.

[9] D. Yang, D. Jung, I. Song, D. Yoo, J. Lee, Comparative investigation into implicit, explicit, and iterative implicit/explicit schemes for the simulation of sheet-metal forming processes, J. Mater. Process. Technol. 50 (1995) 39-53.

[10] J. Gelin, L. Boulmane, P. Boisse, Quasi-static implicit and transient explicit analyses of sheet-metal forming using a $C^{0}$ three-nodes shell element, J. Mater. Process. Technol. 50 (1995) 54-69.

[11] J. Sun, K. Lee, K. Lee, Comparison of implicit and explicit finite element methods for dynamic problem, J. Mater. Process. Technol. 105 (2000) 110-118.

[12] W. Rust, K. Schweizerhof, Finite element limit load analysis of thin-walled structures by ANSYS (implicit), LS-DYNA (explicit) and in combination, Thin-Walled Struct. 41 (2003) 227-244.

[13] J.-P. Ponthot, M. Hogge, On relative merits of implicit schemes for transient problems in metal forming simulation, in: B. Kröplin, E. Luckey (Eds.), International Conference on Numerical Methods for Metal Forming in Industry, vol. 2, Baden-Baden, Germany, 1994, pp. 128-148. 
[14] M. Hogge, J.-P. Ponthot, Efficient implicit schemes for transient problems in metal forming simulation, in: NUPHYMAT'96, Numerical and Physical Study of Material Forming Processes (CEMEF-Ecole nationale supèrieure des mines de Paris SophiaAntipolis, France, 1996).

[15] M. Finn, P. Galbraith, L. Wu, J. Hallquist, L. Lum, T.-L. Lin, Use of a coupled explicit-implicit solver for calculating spring-back in automotive body panels, J. Mater. Process. Technol. 50 (1995) 395-409.

[16] N. Narkeeran, M. Lovell, Predicting springback in sheet metal forming: An explicit to implicit sequential solution procedure, Finite Elements Anal. Des. 33 (1999) 29-42.

[17] L. Noels, L. Stainier, J.-P. Ponthot, J. Bonini, Combined implicit-explicit algorithms for non-linear structural dynamics, Revue Eur. des Elements-Finis 11 (2002) 565-591.

[18] L. Noels, L. Stainier, J.-P. Ponthot, Combined implicit/explicit algorithms for crashworthiness analysis, Int. J. Impact Engrg.

[19] L. Noels, L. Stainier, J.-P. Ponthot, Combined implicit/explicit time integration algorithms for the numerical simulation of sheet metal forming, J. Comput. Appl. Math. 168 (2004) 331-339.

[20] J. Simo, N. Tarnow, The discrete energy-momentum method. Conserving algorithms for nonlinear elastodynamics, J. Appl. Math. Phys. (ZAMP) 43 (1992) 757-792.

[21] D. Jung, D. Yang, Step-wise combined implicit-explicit finite-element simulation of autobody stamping process, J. Mater. Process. Technol. 83 (1998) 245-260.

[22] D. Flanagan, T. Belytschko, Eigenvalues and stable time steps for the uniform strain hexahedron and quadrilateral, J. Appl. Mech. 51 (1984) 35-40.

[23] L. Noels, L. Stainier, J.-P. Ponthot, Self-adapting time integration management in crash-worthiness and sheet metal forming computations, Int. J. Vehicle Des. 30 (2) (2002) 1-48.

[24] W. Daniels, A partial velocity approach to subcycling structural dynamics, Comput. Methods Appl. Mech. Engrg. 192 (2003) 375394.

[25] J. Simo, N. Tarnow, K. Wong, Exact energy-momentum conserving algorithms and sympletic schemes for nonlinear dynamics, Comput. Methods Appl. Mech. Engrg. 100 (1992) 63-116.

[26] O. Gonzalez, J. Simo, On the stability of sympletic and energy-momentum algorithms for non-linear Hamiltonian systems with symmetry, Comput. Methods Appl. Mech. Engrg. 134 (1996) 197-222.

[27] O. Gonzalez, Mechanical systems subject to holonomic constraints: Differential-algebraic formulations and conservative integration, Physica D 132 (1999) 165-174.

[28] J. Simo, O. Gonzalez, Recent results on the numerical integration of infinite-dimensional Hamiltonian system, in: T. Hughes, E. Onate, O. Zienkiewicz (Eds.), Recent Developments in Finite Element Analysis, CIMNE, Barcelona, Spain, 1994, pp. $255-271$.

[29] T. Laursen, X. Meng, A new solution procedure for application of energy-conserving algorithms to general constitutive models in nonlinear elastodynamics, Comput. Methods Appl. Mech. Engrg. 190 (2001) 6309-6322.

[30] O. Gonzalez, Exact energy and momentum conserving algorithms for general models in nonlinear elasticity, Comput. Methods Appl. Mech. Engrg. 190 (2000) 1763-1783.

[31] X. Meng, T. Laursen, Energy consistent algorithms for dynamic finite deformation plasticity, Comput. Methods Appl. Mech. Engrg. 191 (2001) 1639-1675.

[32] X. Meng, T. Laursen, On energy consistency of large deformation plasticity models, with application to the design of unconditionally stable time integrators, Finite Elements Anal. Des. 38 (2002) 949-963.

[33] L. Noels, L. Stainier, J.-P. Ponthot, Energy-momentum conserving algorithm for non-linear hypoelastic constitutive models, Int. J. Numer. Methods Engrg. 59 (2004) 83-114.

[34] L. Noels, L. Stainier, J.-P. Ponthot, On the use of large time steps with an energy-momentum conserving algorithm for non-linear hypoelastic constitutive models, Int. J. Solids Struct. 41 (2004) 663-693.

[35] J.-P. Ponthot, Unified stress update algorithms for the numerical simulation of large deformation elasto-plastic and elastoviscoplastic processes, Int. J. Plasticity 18 (2002) 91-126.

[36] M. Wilkins, Calculation of elastoplastic flows, in: B. Alder (Eds.), Methods of Comput. Phys., 1964, pp. 211-263 (Chapter 3).

[37] M. Maenchen, S. Sack, The Tensor code, in: B. Alder (Eds.), Methods of Comput. Phys., 1964, pp. 387-400 (Chapter 3).

[38] J. Simo, T. Hughes, Computational Inelasticity, Springer, 1998.

[39] F. Armero, I. Romero, On the formulation of high-frequency dissipative time-stepping algorithms for non-linear dynamics. Part I: Low-order methods for two model problems and nonlinear elastodynamics, Comput. Methods Appl. Mech. Engrg. 190 (2001) 2603-2649.

[40] F. Armero, I. Romero, On the formulation of high-frequency dissipative time-stepping algorithms for non-linear dynamics. Part II: Second-order methods, Comput. Methods Appl. Mech. Engrg. 190 (2001) 6783-6824. 